\title{
Phase behavior and microstructural length scales of a diblock copolymer in the presence of a selective solvent
}

\author{
Ching-I Huang*, Hsiao-Yang Hsueh \\ Institute of Polymer Science and Engineering, National Taiwan University, No. 1, Roosevelt Road, Sec. 4, Taipei 106, Taiwan
}

Received 19 May 2006; received in revised form 10 July 2006; accepted 15 July 2006

Available online 14 August 2006

\begin{abstract}
We employ self-consistent mean-field (SCMF) theory in studying the phase behavior as well as the microstructural domain sizes for a diblock copolymer in the presence of a selective solvent. First we examine the effects of solvent addition on the formation of fcc and bcc packed spheres. As has been found in experiments, the so-called "normal" spheres, i.e., formed by the minority blocks, tend to pack into the bcc array, while the "inverted" spheres formed by the majority blocks favor the fcc packing. Upon increasing the solvent selectivity and/or solvent amount, the formed inverted spheres tend to pack from bcc to fcc. This thermotropic transition of bcc $\rightarrow$ fcc upon increasing the solvent selectivity is induced by the fact that the intermicellar interactions vary from long-range to short-range via a combination of the solvent exclusion from the cores and an increase in the aggregation number. In analyzing the effects of solvent addition on the microstructural sizes, the SCMF results have successfully captured the crossover behavior of characteristic domain spacing from decreasing with added solvent to increasing by increasing the solvent selectivity. Further, the variation of the characteristic domain spacing when the systems transform to a more curved structure changes from a discontinuous decreasing behavior to even a discontinuous increasing behavior upon increasing the solvent selectivity and/or the formation of inverted structures.
\end{abstract}

(C) 2006 Elsevier Ltd. All rights reserved.

Keywords: Self-consistent mean-field theory; Block copolymer solutions; Solvent selectivity

\section{Introduction}

Block copolymers, because of the immiscibility of the covalently bonded blocks, can form a rich variety of morphologies [1-3]. These self-assembled ordered structures typically range between 1 and $100 \mathrm{~nm}$ in size, and have valuable nanotechnological applications. One of the major methods to control the structural type as well as the length scale of these microstructures is by diluting a diblock copolymer with solvent. When a solvent $\mathrm{S}$ is added to an $\mathrm{AB}$ diblock copolymer, its selfassembling behaviors become more diverse, a result of the interplay of the effects of degree of copolymerization $N$, copolymer composition $f\left(=N_{\mathrm{A}} /\left(N_{\mathrm{A}}+N_{\mathrm{B}}\right)\right)$, copolymer volume

\footnotetext{
* Corresponding author. Tel.: +886 2 33665883; fax: +8862 33665237 . E-mail address: chingih@ntu.edu.tw (C.-I. Huang).
}

fraction $\phi$, and three independent interaction parameters, $\chi_{\mathrm{AB}}$, $\chi_{\mathrm{AS}}$, and $\chi_{\mathrm{BS}}$.

When neutral (i.e., $\chi_{\mathrm{AS}}=\chi_{\mathrm{BS}}$ ), provided the concentrated regime and the solvent quality is good, previous self-consistent mean-field (SCMF) calculations have shown that the equilibrium solution phase maps are almost identical to the melt phase map by replacing $\chi_{\mathrm{AB}} N$ with $\phi \chi_{\mathrm{AB}} N$ [4-7], the socalled "dilution approximation" [8]. In that case, the factors governing the phase diagram are simply the copolymer composition $f$ and $\phi \chi_{\mathrm{AB}} N$. As in the melts, the composition $f$ largely determines the geometry of the microstructure, in which the shorter blocks form the minor-domains [7]. In the semi-dilute regime due to the chain swelling effects, both Olvera de la Cruz [9] and Fredrickson and Leibler [10] predicted $\left(\phi^{1.59} \chi_{\mathrm{AB}} N\right)_{\mathrm{ODT}}=F(f)$, i.e., the dilution approximation fails. Naughton and Matsen [11] have employed SCMF theory to examine the accuracy of the dilution approximation as a 
function of solvent quality, size, and selectivity. We further analyzed the dependence of the microstructural length scales with copolymer volume fraction, the interaction parameter, and degree of copolymerization, which indeed correlates very well with the non-uniformity degree of the neutral solvent along the interfaces [12]. Although experiments have shown that the dilution approximation fails to describe the orderdisorder transition (ODT) even for the concentrated block copolymer solutions due to the fluctuation effects [13-16], it is still successful in predicting the order-order transitions (OOTs) [14-16] and microdomain spacings [14-18].

When a selective solvent is added (i.e., $\chi_{\mathrm{AS}} \neq \chi_{\mathrm{BS}}$ ), the shape and the packing symmetry of the ordered structure are determined not only by the composition $f$ but also by the solvent selectivity. There have been a great deal of experimental $[14,15,19-32]$ and theoretical $[7,15,33,34]$ studies on the resulting phase behavior and microstructural length scales. From the theoretical standpoint, Banaszak and Whitmore [33] first employed SCMF theory to examine the lamellar microdomain spacing as a function of the copolymer volume fraction $\phi$, degree of copolymerization, and solvent selectivity. They found that upon dilution of a selective solvent but still good for both blocks, the domain spacing decreases as $D_{\mathrm{P}}^{*} \sim \phi^{\alpha}$ with $\alpha$ equal to 0.2 in the weak segregation regime and 0.5 in the strong segregation regime. However, they did not consider a strongly selective solvent in which one block is insoluble. Hanley et al. [15] therefore employed SCMF theory to analyze the lamellar domain spacing as a function of $\phi$ by varying solvent selectivity. They found that the power-law exponent $\alpha$ decreases from $1 / 3$ in the neutral solvent to even a negative value as the solvent becomes poorer for one block. This reflects that the addition of a strongly selective solvent to diblock copolymers is no longer a diluent effect, but instead, enhances the effective segregation between both blocks. Although this crossover behavior of the dependence of domain spacing on copolymer volume fraction by varying the solvent selectivity has also been observed in experiments [15,20,32], the solvent effects on the behavior for each segregated domain spacing within the same ordered phase as well as across the OOTs remain unexplored theoretically. Later we will show that this detailed analysis of each domain length is instructive to understand the mechanism of a thermoreversible fcc $\rightarrow$ bcc transition. Recall that when the diblock copolymer melts undergo an order-order transition into a more curved microstructure, the characteristic domain spacing exhibits a discontinuously decreasing behavior, which results in allowing the larger blocks to relax at the expense of the smaller blocks [35]. When a solvent is added, Hanley et al. [15] reported that in addition to the discontinuously decreasing behavior, the resulting domain spacing accompanying the OOTs exhibits a continuously or even discontinuously increasing behavior.

In analyzing the effects of solvent addition on the phase behavior, Huang and Lodge [7] presented a numerical phase behavior for an $\mathrm{AB}$ diblock copolymer in the presence of a solvent based on the SCMF theory. They examined the effects of solvent selectivity, $\phi, f$, and $N$. Although they only considered the classical phases, including lamellae (L), hexagonally packed cylinders (C), and body-centered cubic spheres $\left(\mathrm{S}^{\mathrm{BCC}}\right)$, they observed that both solvent selectivity and $\phi$ strongly influence the morphology. In contrast to the neat diblock copolymer microstructures in which the shorter blocks form the minor-domains, block copolymer solutions may form the "inverted" phases, where the longer blocks form the minor-domains, by varying solvent selectivity and $\phi$. These theoretical results have also been captured in experiments. For example, Lodge et al. [15,27] have constructed the phase diagrams for poly(styrene- $b$-isoprene) (PS-PI) diblock copolymers in the presence of a solvent, which ranges from slightly to strongly selective for PS blocks. In addition to the various normal and inverted structures, they found that coexistence between lamellae and cylinders is more stable than the gyroid phase at stronger segregations induced by the solvent selectivity. They also observed the existence of facecentered cubic spheres $\left(\mathrm{S}^{\mathrm{FCC}}\right)$, which however, have been ignored in our previous SCMF analysis [7]. They reported that the fcc packing of the spheres formed by the majority blocks is more stable than bcc with increasing solvent selectivity and decreasing copolymer volume fraction $\phi$.

Indeed, the spherical packing order in diblock copolymer solutions has been extensively studied in experiments [15, 19-21,24-30]. Watanabe et al. [19] and Shibayama et al. [20] first showed that with the addition of a PB-selective solvent tetradecane, poly(styrene- $b$-butadiene) (PS-PB) diblock copolymers transform from hexagonally packed PS-cylinders in the bulk to bcc packed PS-spheres. Later, McConnell et al. [21,24] observed that for PS-PI diblocks in the presence of a PI-selective solvent decane, the formed spheres with thicker corona layers, i.e., the so-called "normal" spheres, have long-ranged interactions, and thus adopt a less dense bcc packing like the soft spheres; while for the spheres with thinner corona layers (i.e., "inverted" spheres), the intermicellar interactions become short-ranged and thus like the hard spheres they favor a more dense packing of fcc. A schematic plot of the normal and inverted micelles which prefer a bcc and fcc packing, respectively, is shown in Fig. 1. Their conclusions have captured the thermotropic transition from bcc to fcc spheres for PEO-PPO diblock copolymers in aqueous solutions by Hamley and coworkers $[25,26]$ and most of the observations for PS-PI in various solvents [15,27] by Lodge group. However, Lodge and coworkers reported that a bcc packing is still possible for the inverted spheres with thinner corona layers. A thermoreversible fcc $\rightarrow$ bcc transition occurs upon decreasing the solvent selectivity.

In this paper, we employ SCMF theory to study the spherical bcc versus fcc packing order and the microstructural sizes of an $\mathrm{AB}$ diblock copolymer in the presence of a selective solvent $\mathrm{S}$. In particular, the effects of solvent selectivity, copolymer volume fraction $\phi$, and copolymer composition $f$ are examined. We construct the phase diagrams by comparing the free energies of $\mathrm{L}, \mathrm{C}, \mathrm{S}^{\mathrm{BCC}}$, and $\mathrm{S}^{\mathrm{FCC}}$, which has been ignored in our previous work [7]. Other possible ordered phases such as the gyroid and disordered micelles are not examined here. In addition to the ordered microphases, the systems with lower values of copolymer volume fraction $\phi$ when the 

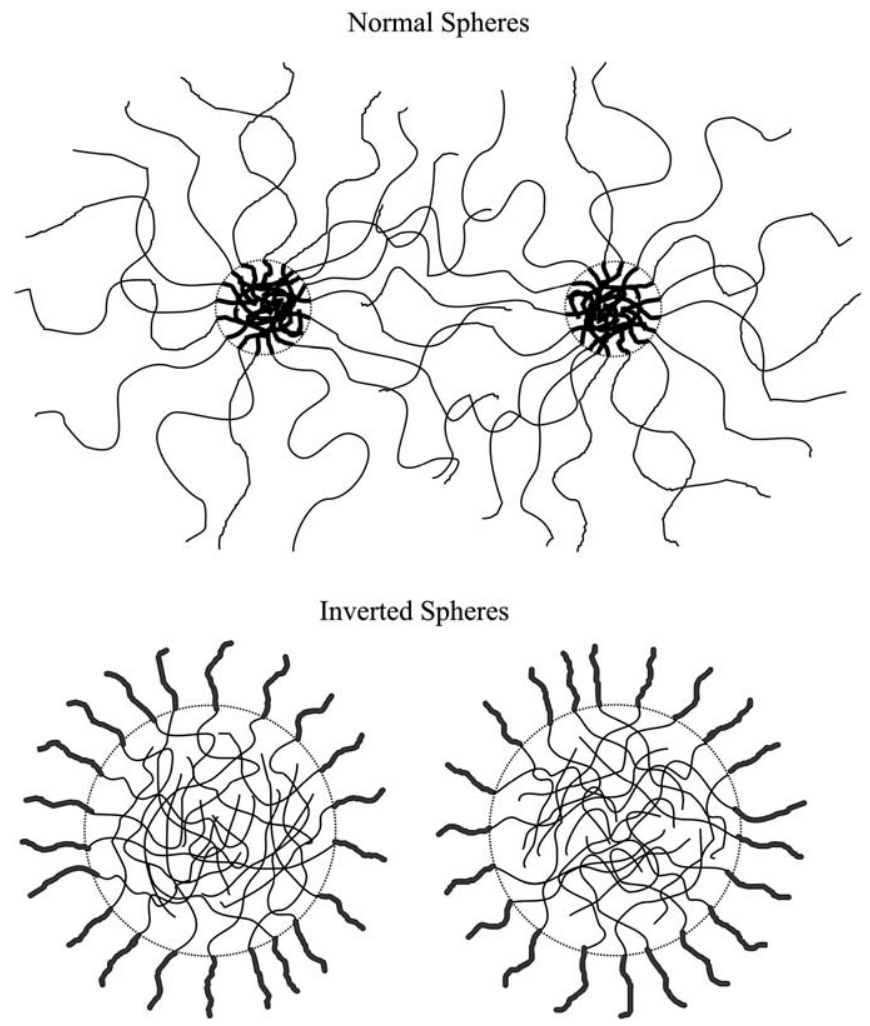

Fig. 1. Schematic illustration of the normal spheres and inverted spheres, which prefer bcc and fcc ordering, respectively.

solvent selectivity is large may undergo a macrophase separation into two phases rich in the solvent and diblock copolymer, respectively, which however, is not our current concern. We thus use the Flory-Huggins thermodynamic analysis to locate the binodal coexistence curve between 2 disordered phases, for simplicity. We then analyze how the added solvent affects the related microstructural domain length scales, such as the characteristic domain spacing, the minor-domain width, the interfacial width, and the matrix (major-domain) length. In particular, the variation of each segregated domain size within the same ordered phase as well as across the order-order transitions is analyzed in detail.

\section{Theory}

\subsection{Self-consistent mean-field theory}

We employ self-consistent mean-field (SCMF) theory to examine the phase behavior and analyze the structural length scales in the diblock copolymer solutions, by using a previously established formalism [7]. We consider a monodisperse $\mathrm{AB}$ diblock copolymer in the presence of a solvent with average volume fractions $\phi$ and $1-\phi$. The degree of copolymerization is $N$ and A-monomer fraction in the copolymer is $f$. We assume that the system is incompressible both locally and globally, and each monomer type has the same statistical segment length $b$. The local interaction between each pair of monomers $\mathrm{I}$ and $\mathrm{J}$, is quantified by the Flory-Huggins interaction parameter $\chi_{\mathrm{IJ}}$. Each copolymer chain is parameterized by a variable $s$ that increases continuously from 0 to 1 along its length. We assume that the A-block starts from $s=0$ and terminates at $s=f$ which is the $\mathrm{A}-\mathrm{B}$ junction point.

In order to determine the concentration profiles as well as the free energy in the equilibrium state, we begin with solving the copolymer partial partition functions, $q_{\mathrm{C}}(r, s)$ and $q_{\mathrm{C}}^{+}(r, s)$, which satisfy the modified diffusion equation,

$\frac{\partial q_{\mathrm{C}}}{\partial s}=\left\{\begin{array}{lll}\frac{1}{6} N b^{2} \nabla^{2} q_{\mathrm{C}}-\omega_{\mathrm{A}} q_{\mathrm{C}} & \text { if } & s<f \\ \frac{1}{6} N b^{2} \nabla^{2} q_{\mathrm{C}}-\omega_{\mathrm{B}} q_{\mathrm{C}} & \text { if } & s>f\end{array}\right.$

and the initial condition is $q_{\mathrm{C}}(r, 0)=1$. The equation for $q_{\mathrm{C}}^{+}(r, s)$ is similar except that the right-hand side of Eq. (1) is multiplied by -1 , and the initial condition is $q_{\mathrm{C}}^{+}(r, 1)=$ 1. In Eq. (1), $\omega_{\mathrm{A}}(r)$ and $\omega_{\mathrm{B}}(r)$ represent the fields acting on the A segments and B segments along the copolymer chains from $s=0$ to $f$ and from $s=f$ to 1 , respectively. The total partition function for a single copolymer chain $Q_{\mathrm{C}}$ is found by integrating all possible configurations for the chains subject to these fields and thus equal to

$Q_{\mathrm{C}}=\frac{1}{V} \int_{V} q_{\mathrm{C}}(r, s=1) \mathrm{d}^{3} r$

For the solvent particles, the partition function $Q_{\mathrm{S}}$ subject to the field $\omega_{\mathrm{S}}(r)$ is simply equal to

$Q_{\mathrm{S}}=\frac{1}{V} \int_{V} \exp \left[\frac{-\omega_{\mathrm{S}}(r)}{N}\right] \mathrm{d}^{3} r$

which indeed can be rewritten as

$Q_{\mathrm{S}}=\frac{1}{V} \int_{V} q_{\mathrm{S}}\left(r, s=\frac{1}{N}\right) \mathrm{d}^{3} r$

where $q_{\mathrm{S}}(r, s)$ satisfies

$\frac{\partial q_{\mathrm{S}_{1}}}{\partial s}=-\omega_{\mathrm{S}_{1}} q_{\mathrm{S}_{1}}$

with the initial condition $q_{\mathrm{S}}(r, s=0)=1$.

As the diblock copolymer morphologies are periodic, it is most efficient to perform the SCMF calculations using the Fourier-space algorithm. That is, any given function, $g(r)$ is expressed in terms of the corresponding amplitudes, $g_{j}$, with respect to a series of orthonormal basis function $f_{j}(r), g(r)=$ $\sum_{j} g_{j} f_{j}(r)$. The basis functions reflect the symmetry of the ordered phase being considered, and are selected to be eigenfunctions of the Laplacian operator

$\nabla^{2} f_{j}(r)=-\lambda_{j} L^{-2} f_{j}(r)$

where $L$ is the lattice spacing for the ordered phase. The basis functions are ordered starting with $f_{1}(r)=1$ such that $\lambda_{j}$ is an increasing series. For lamellae $f_{j}(r)=2^{1 / 2} \cos (2 \pi(j-1) x / L)$, 
$j \geq 2$, where $x$ is the coordinate orthogonal to the lamellae. Basis functions for the phases with other space-group symmetries can be found in Ref. [36]. Note that the number of basis functions varies with the ordered phase and the segregation degree. In any case, sufficient number of basis functions has to be included in our computations in order to assure that the results reach the equilibrium values.

When the amplitudes corresponding to the basis functions are utilized, Eqs. (1) and (5) for solving $q_{\mathrm{C}}(r, s)$ and $q_{\mathrm{S}}(r, s)$ become

$$
\begin{aligned}
& \frac{\partial q_{\mathrm{C}, i}}{\partial s}=\left\{\begin{array}{ll}
\sum_{j} A_{i j} q_{\mathrm{C}, j} & \text { if } s<f \\
\sum_{j} B_{i j} q_{\mathrm{C}, j} & \text { if } \quad s>f
\end{array} \quad i=1,2,3, \ldots\right.
\end{aligned}
$$

The equation for $q_{\mathrm{C}, i}^{+}$is similar except that the right-hand side of Eq. (7) is multiplied by -1 . The initial conditions are $q_{\mathrm{C}, i}(s=0)=\delta_{i 1}, \quad q_{\mathrm{C}, i}^{+}(s=1)=\delta_{i 1}, \quad$ and $q_{\mathrm{S}, i}(s=0)=\delta_{i 1}$. The matrices $A_{i j}, B_{i j}$, and $C_{i j}$ are given by

$$
\begin{aligned}
A_{i j} & =-\frac{N b^{2}}{6 L^{2}} \lambda_{i} \delta_{i j}-\sum_{k} \omega_{\mathrm{A}, k} \Gamma_{i j k} \\
B_{i j} & =-\frac{N b^{2}}{6 L^{2}} \lambda_{i} \delta_{i j}-\sum_{k} \omega_{\mathrm{B}, k} \Gamma_{i j k} \\
C_{i j} & =-\sum_{k} \omega_{\mathrm{S}, k} \Gamma_{i j k}
\end{aligned}
$$

with $\Gamma_{i j k}=V^{-1} \int f_{i}(r) f_{j}(r) f_{k}(r) \mathrm{d} r . \omega_{\mathrm{A}, k}, \omega_{\mathrm{B}, k}$, and $\omega_{\mathrm{S}, k}$ are the corresponding amplitudes with respect to the $k$ th basis function for fields $\omega_{\mathrm{A}}, \omega_{\mathrm{B}}$, and $\omega_{\mathrm{S}}$, respectively.

Based on the minimization of free energy to attain thermodynamic equilibrium for a periodic ordered phase, the amplitudes of the fields have to satisfy

$$
\begin{aligned}
& \omega_{\mathrm{A}, i}-\omega_{\mathrm{S}, i}=\chi_{\mathrm{AB}} N \phi_{\mathrm{B}, i}+\chi_{\mathrm{AS}} N \phi_{\mathrm{S}, i}-\chi_{\mathrm{AS}} N \phi_{\mathrm{A}, i}-\chi_{\mathrm{BS}} N \phi_{\mathrm{B}, i} \\
& \omega_{\mathrm{B}, i}-\omega_{\mathrm{S}, i}=\chi_{\mathrm{AB}} N \phi_{\mathrm{A}, i}+\chi_{\mathrm{BS}} N \phi_{\mathrm{S}, i}-\chi_{\mathrm{AS}} N \phi_{\mathrm{A}, i}-\chi_{\mathrm{BS}} N \phi_{\mathrm{B}, i} \\
& \phi_{\mathrm{A}, i}+\phi_{\mathrm{B}, i}+\phi_{\mathrm{S}, i}=\delta_{i 1}
\end{aligned}
$$

where the amplitudes of the concentrations of $\mathrm{A}, \mathrm{B}$, and $\mathrm{S}$, respectively, $\phi_{\mathrm{A}, i}, \phi_{\mathrm{B}, i}$, and $\phi_{\mathrm{S}, i}$ are expressed in terms of the distribution functions

$$
\begin{aligned}
\phi_{\mathrm{A}, i} & =\frac{\phi}{q_{\mathrm{C}, 1}(1)} \sum_{j, k} \Gamma_{i j k} \int_{0}^{f} \mathrm{~d} s q_{\mathrm{C}, j}(s) q_{\mathrm{C}, k}^{+}(s) \\
\phi_{\mathrm{B}, i} & =\frac{\phi}{q_{\mathrm{C}, 1}(1)} \sum_{j, k} \Gamma_{i j k} \int_{f}^{1} \mathrm{~d} s q_{\mathrm{C}, j}(s) q_{\mathrm{C}, k}^{+}(s) \\
\phi_{\mathrm{S}, i} & =\frac{1-\phi}{q_{\mathrm{S}, 1}(1 / N)} q_{\mathrm{S}, i}\left(\frac{1}{N}\right)
\end{aligned}
$$

Once the above amplitudes are determined and the selfconsistent equations for the fields are satisfied, the free energy per molecule $F$ is given by

$$
\begin{aligned}
\frac{F}{k_{\mathrm{B}} T}= & -\phi \ln \left[\frac{q_{\mathrm{C}, 1}(1)}{\phi}\right]-(1-\phi) N \ln \left[\frac{q_{\mathrm{S}, 1}(1 / N)}{1-\phi}\right] \\
& -\sum_{i}\left(\omega_{\mathrm{A}, i} \phi_{\mathrm{A}, i}+\omega_{\mathrm{B}, i} \phi_{\mathrm{B}, i}+\omega_{\mathrm{S}, i} \phi_{\mathrm{S}, i}\right) \\
& +\sum_{i}\left(\chi_{\mathrm{AB}} N \phi_{\mathrm{A}, i} \phi_{\mathrm{B}, i}+\chi_{\mathrm{AS}} N \phi_{\mathrm{A}, i} \phi_{\mathrm{S}, i}+\chi_{\mathrm{BS}} N \phi_{\mathrm{B}, i} \phi_{\mathrm{S}, i}\right)
\end{aligned}
$$

For a disordered state, the volume fractions $\phi_{\mathrm{I}}(r)$ and the fields $\omega_{\mathrm{I}}(r)$ are constants, i.e., $\phi_{\mathrm{I}}(r)=\phi_{\mathrm{I}, 1}$ and $\omega_{\mathrm{I}}(r)=\omega_{\mathrm{I}, 1}$, $\mathrm{I}=\mathrm{A}, \mathrm{B}, \mathrm{S}$. The partition functions are simply $Q_{\mathrm{C}}=q_{\mathrm{C}, 1}(1)=$ $\exp \left(-f \omega_{\mathrm{A}, 1}-(1-f) \omega_{\mathrm{B}, 1}\right)$ and $Q_{\mathrm{S}}=q_{\mathrm{S}, 1}(1 / N)=\exp$ $\left(-\omega_{\mathrm{S}, 1} / N\right)$. As a result, Eq. (12) is reduced to the Flory-Huggins mean-field free energy functional per molecule in the disordered state, i.e.,

$$
\begin{aligned}
\frac{F}{k_{\mathrm{B}} T_{\text {disorder }}}= & \phi \ln \phi+(1-\phi) N \ln (1-\phi)+f(1-f) \chi_{\mathrm{AB}} N \phi^{2} \\
& +f \chi_{\mathrm{AS}} N \phi(1-\phi)+(1-f) \chi_{\mathrm{BS}} N \phi(1-\phi)
\end{aligned}
$$

For a periodic ordered phase, the free energy has to be minimized with respect to the lattice spacing $L$; to determine the most stable phase one compares free energies of different possible phases. Since one of our objectives is to address the issue of spherical bcc versus fcc packing order, we restrict consideration to the classical phases, such as lamellae (L), hexagonally packed cylinders $(\mathrm{C})$, body-centered cubic ordered spheres $\left(\mathrm{S}^{\mathrm{BCC}}\right)$, and face-centered cubic ordered spheres $\left(\mathrm{S}^{\mathrm{FCC}}\right)$.

In order to illustrate the variation of the structural length scales in various ordered structures, we first define a characteristic domain spacing $D_{\mathrm{P}}=2 \pi / q_{1}$, where $q_{1}$ is the principal scattering vector for a given structure. As such, $D_{\mathrm{P}}$ is equal to $L, \sqrt{3} L / 2, L / \sqrt{2}$, and $L / \sqrt{3}$, for the phases $\mathrm{L}, \mathrm{C}, \mathrm{S}^{\mathrm{BCC}}$, and $\mathrm{S}^{\mathrm{FCC}}$, respectively. We then divide the microstructure into the minor-domain, major-domain (matrix), and the interface. For ordered cylindrical and spherical phases, the minordomains are simply defined as the cylinders and spheres, respectively. Hence, the minor-domain width $D$ corresponds to the diameter of the cylinders or spheres. The major-domain width $\Lambda$ is defined as the shortest distance between two cylinders (or spheres) minus $D$ and the interfacial width $W$. For the stable L phase, we define the regimes rich in the minority blocks and majority blocks, as the minor-domains and major-domains, respectively. The determination of $D, W$, and $\Lambda$, is given as the following example. When a neutral solvent $\mathrm{S}\left(\chi_{\mathrm{AS}}=\chi_{\mathrm{BS}}=0.4\right)$ is added into a diblock copolymer with $f=0.16, N=300, \phi=0.8$, and $\chi_{\mathrm{AB}} N=41.4, \mathrm{~S}_{\mathrm{A}}^{\mathrm{BCC}}$ is the most stable phase. Fig. 2 shows the typical volume fraction profiles $\phi_{\mathrm{A}}, \phi_{\mathrm{B}}$, and $\phi_{\mathrm{S}}$, at $y / L=z / L=0.5$, from which the inflection points with respect to $\phi_{\mathrm{A}}$ and $\phi_{\mathrm{B}}$; i.e., $\mathrm{d}^{2} \phi_{\mathrm{I}} / \mathrm{d} x^{2}=0(\mathrm{I}=\mathrm{A}$, $\mathrm{B}, \mathrm{S})$ are determined. Note that these inflection points are identical. As such, both values of the minor-domain width $D$ and the interfacial width $W$ are determined, as shown in Fig. 2. The major-domain width $\Lambda$ is thus equal to $(\sqrt{3} / 2) L\left(=\sqrt{3 / 2} D_{\mathrm{P}}\right)-D-W$ for $\mathrm{S}^{\mathrm{BCC}}$ phase. For other 


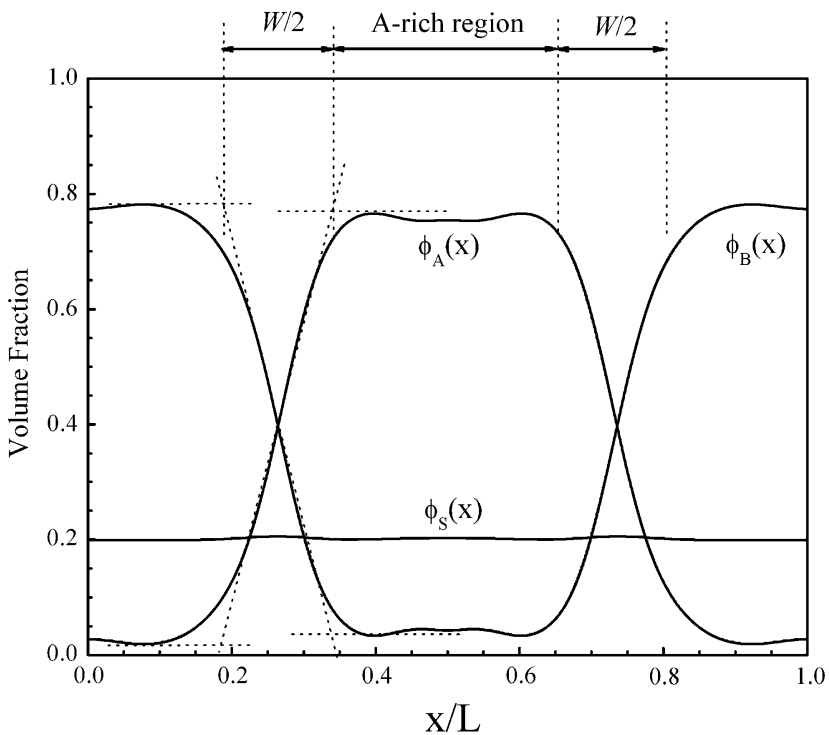

Fig. 2. Volume fraction profiles of $\phi_{\mathrm{A}}, \phi_{\mathrm{B}}$, and $\phi_{\mathrm{S}}$ at $y / L=z / L=0.5$ for diblock copolymer solutions in the $\mathrm{S}_{\mathrm{A}}^{\mathrm{BCC}}$ phase with $f=0.16, N=300$, $\phi=0.8, \chi_{\mathrm{AB}} N=41.4$, and $\chi_{\mathrm{AS}}=\chi_{\mathrm{BS}}=0.4$.

microstructures, similar volume fraction profiles as in Fig. 2 within the bcc spherical phase can also be obtained, from which both values of $D$ and $W$ are determined. As such, $A$ equals $L\left(=D_{\mathrm{P}}\right)-D-W, L\left(=\sqrt{4 / 3} D_{\mathrm{P}}\right)-D-W$, and $(\sqrt{2} / 2) L\left(=\sqrt{3 / 2} D_{\mathrm{P}}\right)-D-W$ for $\mathrm{L}, \mathrm{C}$, and $\mathrm{S}^{\mathrm{FCC}}$, respectively.

Once the micro-domains are divided into distinct minor, interfacial, and major regimes, the volume fractions of each component $\mathrm{A}, \mathrm{B}$, and $\mathrm{S}$, which are partitioned into the minor-domains, interfaces, and major-domains, $\phi_{\mathrm{J}}^{(D)}, \phi_{\mathrm{J}}^{(W)}$, and $\phi_{\mathrm{J}}^{(\Lambda)}$, respectively, $\mathrm{J}=\mathrm{A}, \mathrm{B}$, and $\mathrm{S}$, are calculated by the following equations:

$\phi_{\mathrm{J}}^{(D)}=\frac{1}{L} \int_{D} \phi_{\mathrm{J}}(r) \mathrm{d} r$

$\phi_{\mathrm{J}}^{(W)}=\frac{1}{L} \int_{W} \phi_{\mathrm{J}}(r) \mathrm{d} r \quad \mathrm{~J}=\mathrm{A}, \mathrm{B}, \mathrm{S}, \quad$ for $\mathrm{L}$ phase

$\phi_{\mathrm{J}}^{(\Lambda)}=\frac{1}{L} \int_{\Lambda} \phi_{\mathrm{J}}(r) \mathrm{d} r$

and

$\phi_{\mathrm{J}}^{(D)}=\frac{2}{\sqrt{3} L^{2}} \int_{A_{D}} \phi_{\mathrm{J}}(r) \mathrm{d} A$

$\phi_{\mathrm{J}}^{(W)}=\frac{2}{\sqrt{3} L^{2}} \int_{A_{W}} \phi_{\mathrm{J}}(r) \mathrm{d} A \quad \mathrm{~J}=\mathrm{A}, \mathrm{B}, \mathrm{S}, \quad$ for C phase

$\phi_{\mathrm{J}}^{(\Lambda)}=\frac{2}{\sqrt{3} L^{2}} \int_{A_{\Lambda}} \phi_{\mathrm{J}}(r) \mathrm{d} A$

where $A_{D}=\pi(D / 2)^{2}, A_{W}=\pi\left[(D / 2+W / 2)^{2}-(D / 2)^{2}\right]$, $A_{\Lambda}=\left(\sqrt{3} L^{2} / 2\right)-A_{D}-A_{W}$, and
$\phi_{\mathrm{J}}^{(D)}=\frac{1}{L^{3}} \int_{V_{D}} \phi_{\mathrm{J}}(r) \mathrm{d} V$

$\phi_{\mathrm{J}}^{(W)}=\frac{1}{L^{3}} \int_{V_{W}} \phi_{\mathrm{J}}(r) \mathrm{d} V \quad \mathrm{~J}=\mathrm{A}, \mathrm{B}, \mathrm{S}, \quad$ for $\mathrm{S}$ phase

$\phi_{\mathrm{J}}^{(\Lambda)}=\frac{1}{L^{3}} \int_{V_{\Lambda}} \phi_{\mathrm{J}}(r) \mathrm{d} V$

where for $\mathrm{S}_{\mathrm{A}}^{\mathrm{BCC}}$ phase: $V_{D}=2(4 \pi / 3)(D / 2)^{3}, V_{W}=2(4 \pi / 3)$ $\left[(D / 2+W / 2)^{3}-(D / 2)^{3}\right], V_{\Lambda}=L^{3}-V_{D}-V_{W}$, and for $\mathrm{S}_{\mathrm{A}}^{\mathrm{FCC}}$ phase: $V_{D}=4(4 \pi / 3)(D / 2)^{3}, V_{W}=4(4 \pi / 3)\left[(D / 2+W / 2)^{3}-\right.$ $\left.(D / 2)^{3}\right], V_{\Lambda}=L^{3}-V_{D}-V_{W}$.

It is evident that the sum of $\phi_{\mathrm{J}}^{(D)}, \phi_{\mathrm{J}}^{(W)}$, and $\phi_{\mathrm{J}}^{(\Lambda)}$ is equal to the average volume fraction of component $\mathrm{J}\left(\bar{\phi}_{\mathrm{J}}\right)$. Therefore, the relative volume fraction of component $\mathbf{J}$ into each regime is equal to

$\tilde{\phi}_{\mathrm{J}}^{(D)}=\frac{\phi_{\mathrm{J}}^{(D)}}{\left(\phi_{\mathrm{J}}^{(D)}+\phi_{\mathrm{J}}^{(W)}+\phi_{\mathrm{J}}^{(\Lambda)}\right)}=\frac{\phi_{\mathrm{J}}^{(D)}}{\bar{\phi}_{\mathrm{J}}}$

$\tilde{\phi}_{\mathrm{J}}^{(W)}=\frac{\phi_{\mathrm{J}}^{(W)}}{\left(\phi_{\mathrm{J}}^{(D)}+\phi_{\mathrm{J}}^{(W)}+\phi_{\mathrm{J}}^{(\Lambda)}\right)}=\frac{\phi_{\mathrm{J}}^{(W)}}{\bar{\phi}_{\mathrm{J}}} \quad \mathrm{J}=\mathrm{A}, \mathrm{B}, \mathrm{S}$

$\tilde{\phi}_{\mathrm{J}}^{(\Lambda)}=\frac{\phi_{\mathrm{J}}^{(\Lambda)}}{\left(\phi_{\mathrm{J}}^{(D)}+\phi_{\mathrm{J}}^{(W)}+\phi_{\mathrm{J}}^{(\Lambda)}\right)}=\frac{\phi_{\mathrm{J}}^{(\Lambda)}}{\bar{\phi}_{\mathrm{J}}}$

Note that in Section 3 we use the dimensionless length parameters $D_{\mathrm{P}}^{*}, D^{*}, W^{*}$, and $\Lambda^{*}$, which are in terms of the radius of gyration of copolymer chains $(\sqrt{N / 6} b)$, i.e., $D_{\mathrm{P}}^{*}=$ $D_{\mathrm{P}} /(\sqrt{N / 6} b)$ and similar to $D^{*}, W^{*}$, and $\Lambda^{*}$.

\subsection{Binodal coexistence curves between disordered phases}

To determine the coexistence between disordered phases $(\alpha, \beta, \ldots)$ for a monodisperse $\mathrm{AB}$ diblock copolymer $(\mathrm{C})$ in the presence of one solvent $S$, one has to equate the chemical potential of each component $\mathrm{C}(=\mathrm{AB})$ and $\mathrm{S}$ in the possible coexisting phases,

$\mu_{\mathrm{I}}^{(\alpha)}=\mu_{\mathrm{I}}^{(\beta)} \quad \mathrm{I}=\mathrm{C}, \mathrm{S}$

The chemical potential of component $I$ is defined as the change of the total free energy $\Delta F_{\mathrm{o}}$ with respect to the number of component I, $n_{\mathrm{I}}$,

$\mu_{\mathrm{I}}=\frac{\partial \Delta F_{\mathrm{o}}}{\partial n_{\mathrm{I}}}$

$\Delta F_{\mathrm{o}}=F\left(n_{\mathrm{C}}+\frac{n_{\mathrm{S}}}{N}\right)$

where $F$ is the Flory-Huggins mean-field free energy functional per copolymer molecule and has been given in Eq. (13). Recall that 
$\phi=\frac{n_{\mathrm{C}} N}{n_{\mathrm{C}} N+n_{\mathrm{S}}}$

By chain rules,

$\mu_{\mathrm{C}}=\frac{\partial F}{\partial n_{\mathrm{C}}}\left(n_{\mathrm{C}}+\frac{n_{\mathrm{S}}}{N}\right)+F$

$\mu_{\mathrm{S}}=\frac{\partial F}{\partial n_{\mathrm{S}}}\left(n_{\mathrm{C}}+\frac{n_{\mathrm{S}}}{N}\right)+\frac{F}{N}$

where

$\frac{\partial F}{\partial n_{\mathrm{I}}}=\frac{\partial F}{\partial \phi} \frac{\partial \phi}{n_{\mathrm{I}}} \quad \mathrm{I}=\mathrm{C}, \mathrm{S}$

with

$\frac{\partial \phi}{n_{\mathrm{C}}}=\frac{\phi(1-\phi)}{n_{\mathrm{C}}}$

$\frac{\partial \phi}{n_{\mathrm{S}}}=\frac{-\phi(1-\phi)}{n_{\mathrm{S}}}$

Substituting Eqs. (22) and (23) into (21), $\mu_{\mathrm{I}}, \mathrm{I}=\mathrm{C}, \mathrm{S}$, has the form of

$$
\begin{aligned}
& \mu_{\mathrm{C}}=k_{\mathrm{B}} T\{ \ln \phi+(1-\phi)(1-N)+N\left[f \chi_{\mathrm{AS}}+(1-f) \chi_{\mathrm{BS}}\right] \\
&\left.(1-\phi)^{2}+N f(1-f) \chi_{\mathrm{AB}} \phi(2-\phi)\right\} \\
& \mu_{\mathrm{S}}=k_{\mathrm{B}} T\left\{\ln (1-\phi)+\phi\left(1-\frac{1}{N}\right)+\left[f \chi_{\mathrm{AS}}+(1-f) \chi_{\mathrm{BS}}\right]\right. \\
&\left.\phi^{2}-f(1-f) \chi_{\mathrm{AB}} \phi^{2}\right\}
\end{aligned}
$$

\section{Results and discussion}

\subsection{Effects of composition $f$ and copolymer volume fraction $\phi$ on the phase behavior}

In Fig. 3(a) and (b) we present the phase maps in terms of $f$ and $\phi$ for a diblock copolymer in the presence of a selective solvent $\mathrm{S}$ with interaction parameters $\chi_{\mathrm{AS}}=0.7, \chi_{\mathrm{BS}}=0.4$, and $\chi_{\mathrm{AB}} N=40$, for $N=150$ and 300, respectively. As expected, when the solutions are concentrated, the formation of equilibrium morphology is mainly dominated by the composition $f$, similar to that in the diblock copolymer melts. When the solutions become less concentrated, due to the fact that $S$ prefers the B-block and thus acts in a manner that corresponds qualitatively to reducing the A composition, the formation of A-formed cylinders as well as spheres is expected even for $f>0.5$. It is interesting to find that when the ordered fcc packing spheres are included in the SCMF calculations, there always occurs a region of $S^{\mathrm{FCC}}$ between $\mathrm{S}^{\mathrm{BCC}}$ and $\mathrm{D}$ in the phase diagram. When $f$ is small, this ordered $\mathrm{S}_{\mathrm{A}}^{\mathrm{FCC}}$ regime is very narrow, as observed in the diblock copolymer melts. The occurrence of this very narrow $S^{\mathrm{FCC}}$ region

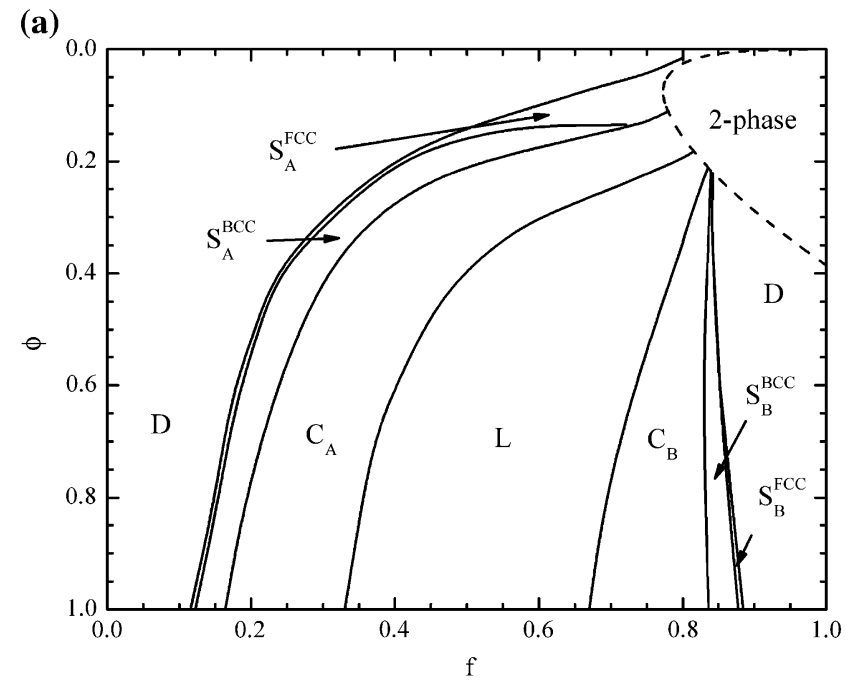

(b)

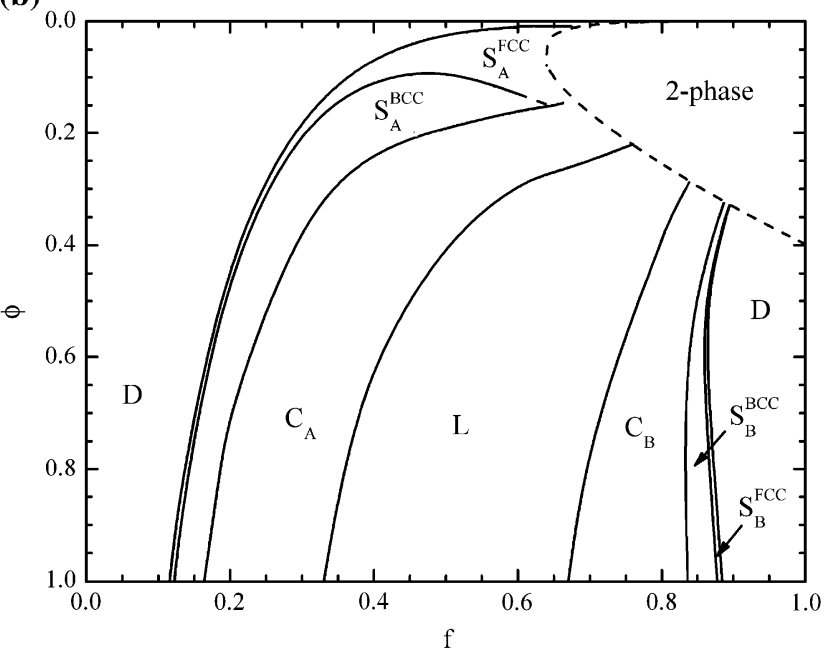

Fig. 3. Two-dimensional phase map as a function of $f$ and $\phi$ for a diblock copolymer solution with $\chi_{\mathrm{AS}}=0.7, \chi_{\mathrm{BS}}=0.4, \chi_{\mathrm{AB}} N=40$, and $N$ equal to (a) 150 and (b) 300 , respectively.

near the ODT for the melts is mainly attributed to the fact that many of the minority blocks are pulled from the spherical domains and thus relieve the packing frustration of the majority blocks in the matrix domains, as in the addition of homopolymers. This enables the spheres to adopt a more dense packing order such as fcc [35]. When a B-selective solvent is added, due to the fact that not only the A-blocks but also the B-selective solvent are pulled from the spheres upon dilution near the ODT, a more dense packing phase of $S^{\mathrm{FCC}}$ is expected too. With increasing the composition of the unfavorable A-blocks, more solvents are expelled into the matrix domains, and thus a greater degree of relieving the packing frustration for the blocks in the matrix is caused. In addition, the corona layers become thinner. As such, this ordered $\mathrm{S}^{\mathrm{FCC}}$ regime near the ODT is greatly expanded with an increase in the composition of unfavorable A-blocks $f$. Recall that in the diblock copolymer melts $S^{\mathrm{FCC}}$ has been recently reported nonexistent in a comparison with the disordered micelle structure [37]. As such, we strongly speculate the stability of this ordered $S^{\mathrm{FCC}}$, 
in particular when the minority block forms the spheres in the solutions. A further examination by comparing the free energy with the disordered micelles will be deferred to a future report. The fact that a bcc ordering is favored over fcc when the minority blocks form the spheres is not surprising since the entropic penalty for larger corona layers is lower in bcc. As $f$ increases to $\geqq 0.5$ so that the majority of A-blocks form the spheres when $\phi$ is small, these spheres can pack into either a bcc or fcc lattice. Further, the stable $\mathrm{S}_{\mathrm{A}}^{\mathrm{BCC}}$ region is reduced and even disappears with an increase in $f$, indicating that the so-called inverted spheres formed by the majority blocks (i.e., with smaller corona layers) prefer a more dense fcc over a bcc packing. As a result, solutions for very asymmetric copolymers with $f \gg 0.5$ may undergo a lyotropic transition of $\mathrm{C}_{\mathrm{A}} \rightarrow \mathrm{S}_{\mathrm{A}}^{\mathrm{FCC}}$ ( $\rightarrow$ Disordered micelles) $\rightarrow \mathrm{D}$; while solutions for approximately symmetric copolymers $(f=0.4-0.6)$ undergo $\mathrm{C}_{\mathrm{A}} \rightarrow \mathrm{S}_{\mathrm{A}}^{\mathrm{BCC}} \rightarrow \mathrm{S}_{\mathrm{A}}^{\mathrm{FCC}}(\rightarrow$ Disordered micelles) $\rightarrow \mathrm{D}$ at moderate values of $\phi$. These theoretical results have been confirmed in experiments [15,21-24,27] except the lyotropic $\mathrm{bcc} \rightarrow \mathrm{fcc}$ transition near the ODT. Note that due to the immiscibility between A-block and solvent, a macrophase separation into an $\mathrm{AB}$ copolymer-rich phase and an S-rich phase occurs when $f>0.5$ and $\psi<1$. As expected, this 2-macrophase regime as well as the 1 ordered microstructure regime is greatly expanded by increasing the degree of copolymerization $N$, as manifested in comparing Fig. 3(a) and (b).

\subsection{Effects of solvent selectivity and $\phi$ on the phase behavior}

To examine the solvent selectivity effects on the stability of fcc and bcc packed spheres, we set $\chi_{\mathrm{BS}}$ equal to 0.4 and vary $\chi_{\mathrm{AS}}$. In Fig. 4(a) and (b), we show the resulting phase map as a function of $\phi$ and $\chi_{\mathrm{AS}}$ for symmetric diblock copolymers ( $f=0.5$ ) with $N=150$ at $\chi_{\mathrm{AB}} N$ equal to 25 and 40 , respectively. As can be seen clearly in each figure, when a slightly selective solvent is added, a sequence from $\mathrm{L} \rightarrow \mathrm{C}_{\mathrm{A}} \rightarrow$ $\mathrm{S}_{\mathrm{A}}^{\mathrm{BCC}} \rightarrow \mathrm{D}$ is observed with decreasing $\phi$. When the selectivity of the added solvent for $\mathrm{B}$ becomes large enough such as $\chi_{\mathrm{AS}}>0.72$ when $\chi_{\mathrm{AB}} N=25$ and $\chi_{\mathrm{AS}}>0.68$ when $\chi_{\mathrm{AB}} N=40$, a transition of $\mathrm{L} \rightarrow \mathrm{C}_{\mathrm{A}} \rightarrow \mathrm{S}_{\mathrm{A}}^{\mathrm{BCC}} \rightarrow \mathrm{S}_{\mathrm{A}}^{\mathrm{FCC}}$ $(\rightarrow$ Disordered micelles $\rightarrow$ D) is expected with increasing dilution. That is, the A-formed spheres in the presence of a slightly B-selective solvent prefer the bcc packing, but they tend to move toward into an fcc array with increasing the solvent selectivity and/or dilution. In a comparison of Fig. 4(a) and (b), we observe that in the slightly selective solvent increasing $\chi_{\mathrm{AB}} N$ enlarges the regime of ordered micro-domains; i.e., the segregation parameter between both A- and B-blocks still has a significant effect on the formation of ordered structures. While, in the strongly selective solvent, the fact that the OOTs seem independent of the value of $\chi_{\mathrm{AB}} N$, reveals that the phase behavior at less concentrated regimes is dominated by the solvent selectivity [15,27].

In reality, varying temperature for a given copolymer solution is often associated with varying $\chi_{\mathrm{AB}}$ and solvent
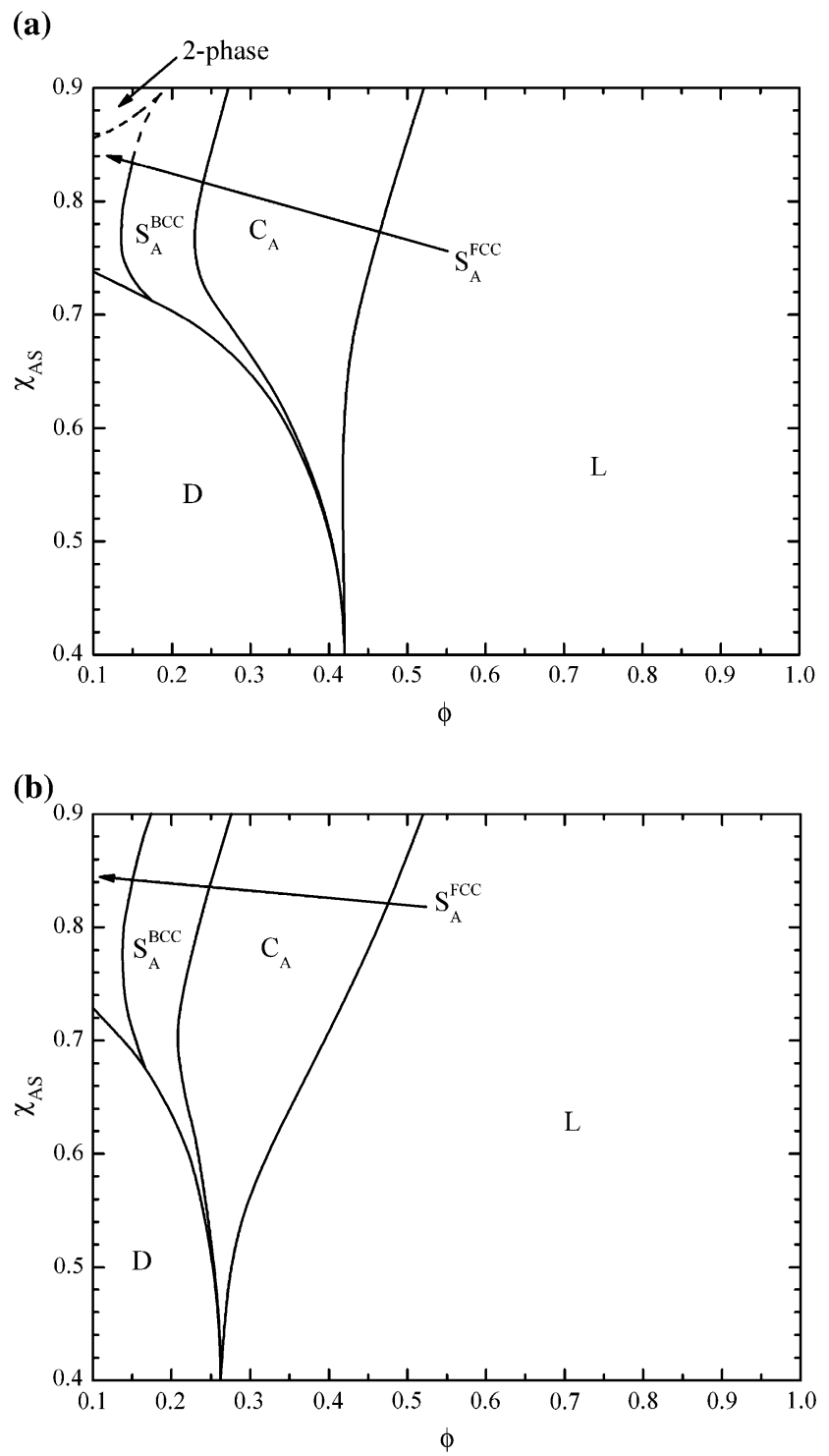

Fig. 4. Two-dimensional phase map as a function of $\phi$ and $\chi_{\mathrm{AS}}$ for a diblock copolymer solution with $f=0.5, N=150, \chi_{\mathrm{BS}}=0.4$, and $\chi_{\mathrm{AB}} N$ equal to (a) 25 and (b) 40, respectively.

selectivity simultaneously. We previously have constructed the two-dimensional phase map as a function of $\chi_{\mathrm{AS}}$ and $\chi_{\mathrm{AB}} N$ for a symmetric diblock copolymer $(f=0.5)$ with $N=200, \chi_{\mathrm{BS}}=0.4$, at $\phi=0.5$ in Ref. [7], which is also presented here as Fig. 5(a). Since we are concerned with the transitions between fcc and bcc by varying temperature, which occur the most for approximately symmetric diblocks at less concentrated solutions, we further construct the resulting phase maps for $f=0.5, N=150, \chi_{\mathrm{BS}}=0.4$, and $\phi$ equal to 0.2 and 0.1 in Fig. 5(b) and (c), respectively. As can be seen clearly in Fig. 5(a) where $\phi=0.5, \mathrm{~L}$ and $\mathrm{C}_{\mathrm{A}}$ are the most stable phases for the interaction parameter and/or temperature regimes examined here. As the copolymer volume fraction decreases to 0.2 (Fig. 5(b)), we find that the ordered L region is significantly compressed and only stable at a very small degree of solvent selectivity. With increasing the solvent 
(a)

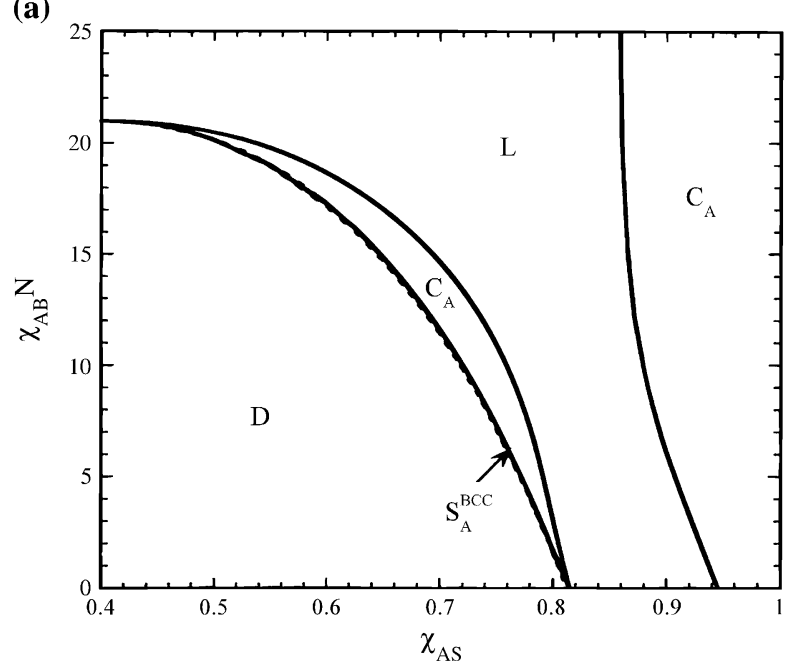

(b)

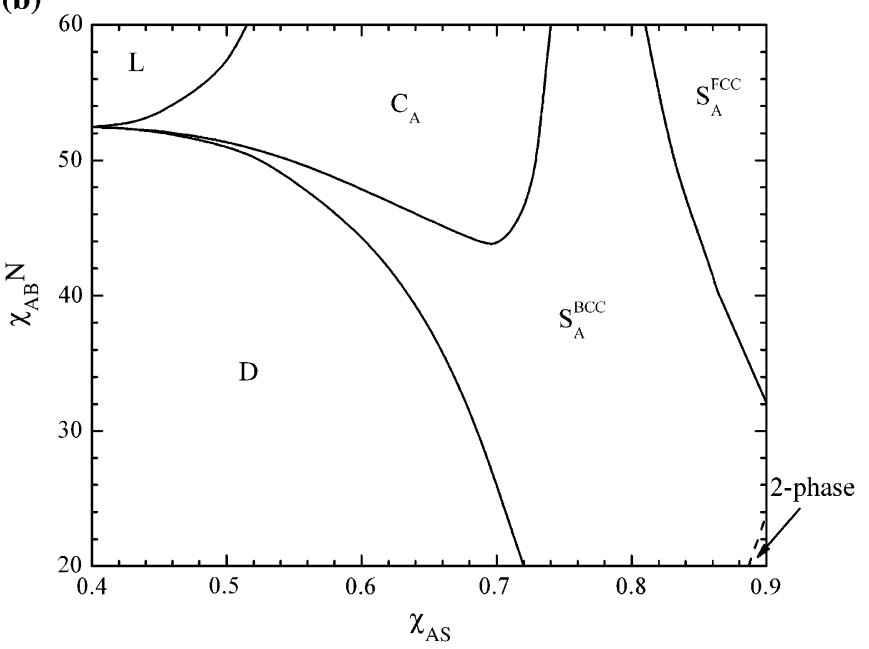

(c)

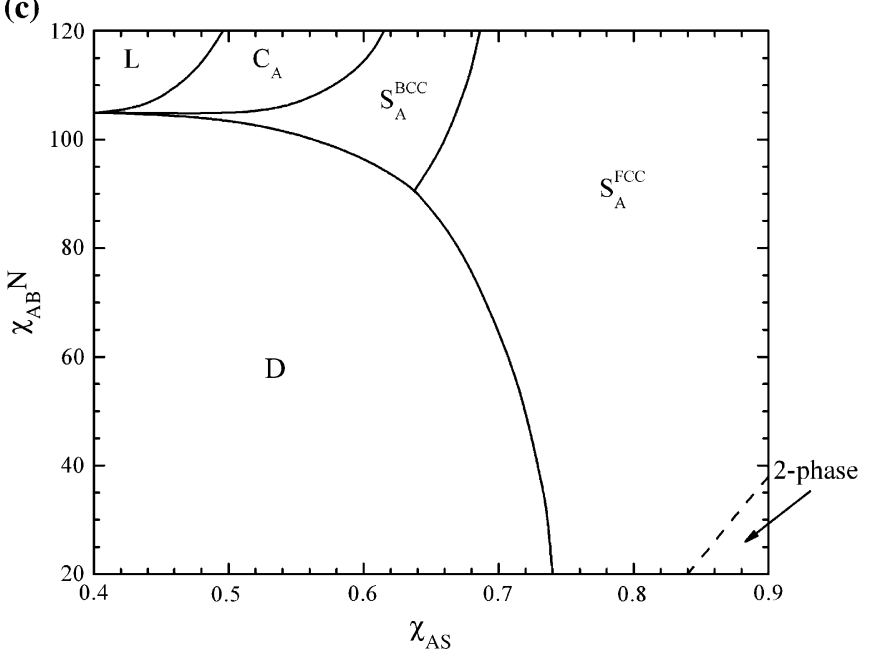

Fig. 5. Two-dimensional phase map as a function of $\chi_{\mathrm{AS}}$ and $\chi_{\mathrm{AB}} N$ for a symmetric diblock copolymer $(f=0.5)$ at a fixed value of $\chi_{\mathrm{BS}}=0.4$ and (a) $N=200, \phi=0.5$, (b) $N=150, \phi=0.2$, and (c) $N=150, \phi=0.1$, respectively. Note that (a) has been presented in Ref. [7]. selectivity, $\mathrm{C}_{\mathrm{A}}$, then $\mathrm{S}_{\mathrm{A}}^{\mathrm{BCC}}$, and then $\mathrm{S}_{\mathrm{A}}^{\mathrm{FCC}}$ become the most stable phases. As a result, various sequential transitions such as $\mathrm{D}$ $\left(\rightarrow \mathrm{S}_{\mathrm{A}}^{\mathrm{BCC}}\right) \rightarrow \mathrm{C}_{\mathrm{A}} \rightarrow \mathrm{S}_{\mathrm{A}}^{\mathrm{BCC}} \rightarrow \mathrm{S}_{\mathrm{A}}^{\mathrm{FCC}}$ or $\mathrm{D} \rightarrow \mathrm{S}_{\mathrm{A}}^{\mathrm{BCC}} \rightarrow \mathrm{S}_{\mathrm{A}}^{\mathrm{FCC}}$ are possible upon decreasing temperature, according to the variation in both $\chi_{\mathrm{AS}}$ and $\chi_{\mathrm{AB}}$ with temperature. With a further decrease of $\phi$ to 0.1 (Fig. 5(c)), there exists a very wide region of ordered $\mathrm{S}_{\mathrm{A}}^{\mathrm{FCC}}$ in the more selective solvent, and a direct transition from $\mathrm{D}$ to $\mathrm{S}_{\mathrm{A}}^{\mathrm{FCC}}$ often occurs upon decreasing temperature. These behaviors have been qualitatively observed in poly(styrene- $b$-isoprene) (PS-PI) diblock copolymers in a series of PS-selective solvents di- $n$-butyl phthalate (DBP), diethyl phthalate (DEP), and dimethyl phthalate (DMP) $[15,27-30]$. The most interesting phenomenon is that both theoretical and experimental studies have confirmed a transition of bcc $\rightarrow$ fcc for the formed spheres of nearly symmetric diblock copolymers in the selective solvent, which is induced by increasing the solvent selectivity. Two possible associated mechanisms via micellar shrinking and an increase in the aggregation number upon increasing the solvent selectivity have been proposed [28]. Through a later detailed analysis of each domain length as well as the distribution of each component, we incline to approve that the transition of bcc $\rightarrow$ fcc upon increasing the solvent selectivity is induced by the fact that the intermicellar interactions vary from long-range to shortrange via a combination of the solvent exclusion from the cores and an increase in the aggregation number.

Lai et al. [31] also examined the phase behavior for PS-PI diblocks but in a very strongly PI-selective solvent such as tetradecane, squalane, and tributylamine. Because of the fact that solvent selectivity in their systems does not change much with temperature, we expect that only a stable phase with appropriate interfacial curvatures exist over a wide range of $\chi_{\mathrm{AB}}$ (i.e., temperature) at a fixed volume fraction $\phi$. As manifested in Fig. 4, if we begin with an ordered lamellar phase for a symmetric diblock copolymer, a series of transitions from $\mathrm{L} \rightarrow$ $\mathrm{C}_{\mathrm{A}} \rightarrow \mathrm{S}_{\mathrm{A}}^{\mathrm{BCC}} \rightarrow \mathrm{S}_{\mathrm{A}}^{\mathrm{FCC}}(\rightarrow$ Disordered micelles $) \rightarrow \mathrm{D}$ is expected by decreasing $\phi$. However, Lai et al. did not observe fcc phase, as we predict here, which we infer may be owing to the fact that they did not extend to less concentrated solutions. Further, whether this fcc phase in more dilute solutions will be replaced by the disordered micelle structure needs to be examined in the future.

\subsection{Effects of solvent selectivity and $\phi$ on the stability of fcc packing normal spheres}

So far, we have elucidated that for nearly symmetric diblock copolymers in the presence of a selective solvent increasing solvent selectivity and/or solvent amount enables the spheres to adopt the fcc packing instead of bcc. Though bcc phase has been reported more stable when the spheres are formed by the minority blocks, we still would like to examine if it is possible for these normal spheres to pack into a fcc lattice by increasing solvent selectivity and/or solvent amount. To examine the effects of a selective solvent on the packing order of the normal spheres formed by the minority 
blocks, we choose a very asymmetric copolymer with $f=0.16$ and $N=600$ in the presence of a B-selective solvent and vary $\chi_{\mathrm{AS}} \geqq \chi_{\mathrm{BS}}=0.4$. Fig. $6(\mathrm{a})-$ (c) presents the two-dimensional phase map in terms of $\chi_{\mathrm{AB}} N$ and $\phi$ when $\chi_{\mathrm{AS}}=0.4,0.7$, and 0.75 , respectively. When a neutral and good solvent $\mathrm{S}\left(\chi_{\mathrm{AS}}=\right.$ $\left.\chi_{\mathrm{BS}}=0.4\right)$ is added to this asymmetric diblock copolymer which forms a $S_{A}^{B C C}$ structure, a sequential lyotropic transition from $\mathrm{S}_{\mathrm{A}}^{\mathrm{BCC}} \rightarrow \mathrm{S}_{\mathrm{A}}^{\mathrm{FCC}} \rightarrow \mathrm{D}$ is expected. Further, both the OOTs and ODT follow the dilution approximation. When the selectivity of the added solvent increases to $\chi_{\mathrm{AS}}=0.7$ (as shown in Fig. 6(b)), though the phase transitions are similar to those in the neutral solutions, and the $\chi_{\mathrm{AB}} N$ values at the ODT and OOTs increase as $\phi$ decreases, we observe that both ordered regimes of $S_{A}^{B C C}$ and $S_{A}^{F C C}$ are enlarged slightly. With a further increase of $\chi_{\mathrm{AS}}$ to 0.75 (Fig. 6(c)), because of the great solvent selectivity, the addition of $\mathrm{S}$ to the $\mathrm{AB}$ diblock copolymers is no longer a diluent effect, but instead, enhances the effective segregation between $\mathrm{A}$ and $\mathrm{B}$. Hence, the $\chi_{\mathrm{AB}} N$ values at the ODT and OOTs first show a decreasing trend and then a very sharp increasing behavior as $\phi$ decreases. In addition to great expansion of the ordered $S_{A}^{B C C}$ region, we also observe that $\mathrm{S}_{\mathrm{A}}^{\mathrm{FCC}}$ is significantly broadened. This is not surprising since increasing solvent selectivity for B enables more solvent to move into the matrix domains rich in the majority of Bblocks, which thus relieves the packing frustration of B. Accordingly, more dense fcc-packed normal spheres are favored at lower values of $\phi$ and larger solvent selectivity. Further, a lyotropic transition of $\mathrm{D} \rightarrow \mathrm{S}_{\mathrm{A}}^{\mathrm{FCC}} \rightarrow \mathrm{D}$ occurs at smaller values of $\chi_{\mathrm{AB}} N$. However, these results based on the current SCMF techniques ignore the possible state of the disordered micelles. To address this issue, a further comparison with the disordered micelle structure remains necessary.

\subsection{Analysis of microstructural length scales}

Varying the copolymer composition, solvent selectivity, and solvent amount, holds great influence not only on the phase behavior but also on the microstructural length scales of diblock copolymer solutions. Fig. 7(a)-(d) presents the variation in characteristic domain spacing $D_{\mathrm{P}}^{*}$, minor-domain width $D^{*}$, interfacial width $W^{*}$, and major-domain width $\Lambda^{*}$, which are in terms of $\sqrt{N / 6} b$, with changes in composition $f$ for a diblock copolymer with $N=150$ and $\chi_{\mathrm{AB}} N=40$ in the presence of a B-selective solvent $\mathrm{S}\left(\chi_{\mathrm{AS}}=0.7, \chi_{\mathrm{BS}}=\right.$ 0.4 ) for $\phi=0.8$ and 0.4. In Fig. 7, we also denote the corresponding equilibrium phase with $f$. Generally, when the solutions are concentrated $(\phi=0.8)$ so that the solvent selectivity effect is not significant, each length parameter is more or less symmetric about $f=0.5$. That is, the composition $f$ dominates the formation of the equilibrium microstructures. The so-called minor-domains and the major-domains are mainly formed by the minority blocks and the majority blocks, respectively. As in the melts [35], because of the fact that increasing the asymmetry degree in $f$ is analogous to reducing the segregation degree, $W^{*}$ increases, and $D_{\mathrm{P}}^{*}, D^{*}$, and $\Lambda^{*}$, decrease until when the ODT is approached, there is a sharp increase
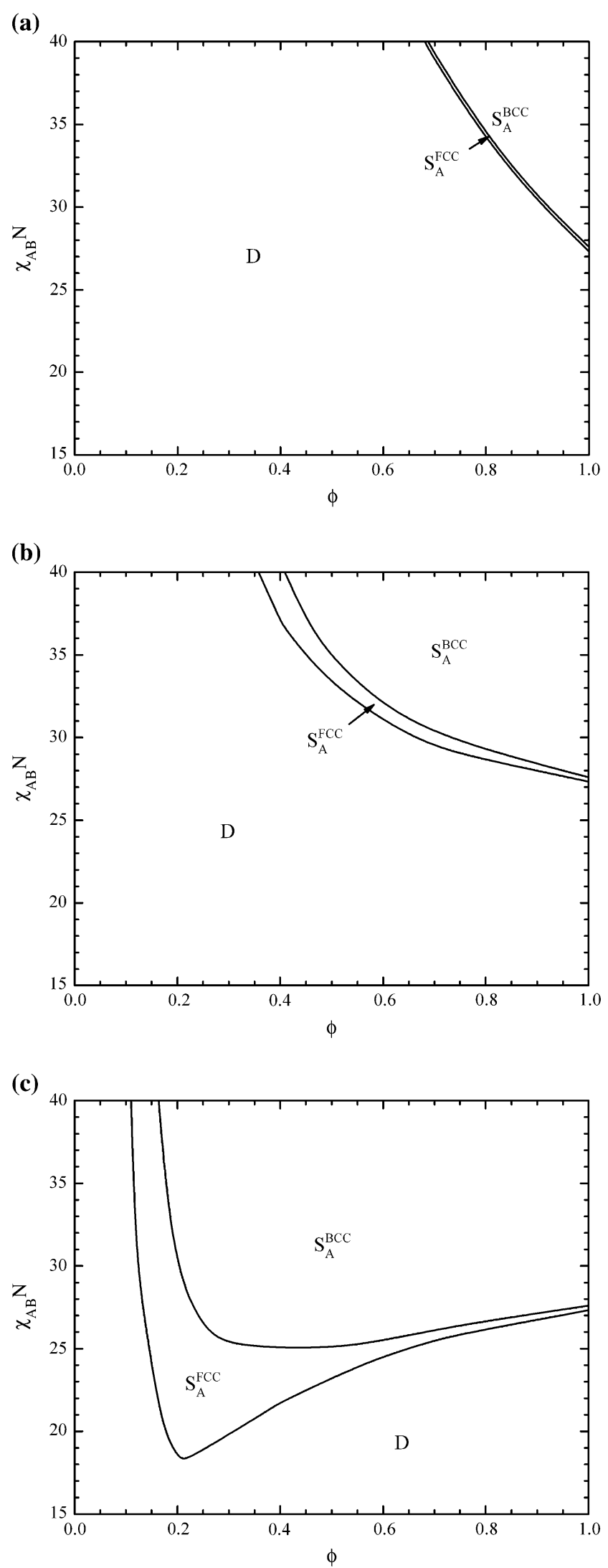

Fig. 6. Two-dimensional phase map in terms of $\phi$ and $\chi_{\mathrm{AB}} N$ for a diblock copolymer with $f=0.16$ and $N=600$ at a fixed value of $\chi_{\mathrm{BS}}=0.4$ and $\chi_{\mathrm{AS}}$ equal to (a) 0.4 , (b) 0.7 , and (c) 0.75 , respectively. 
(a)

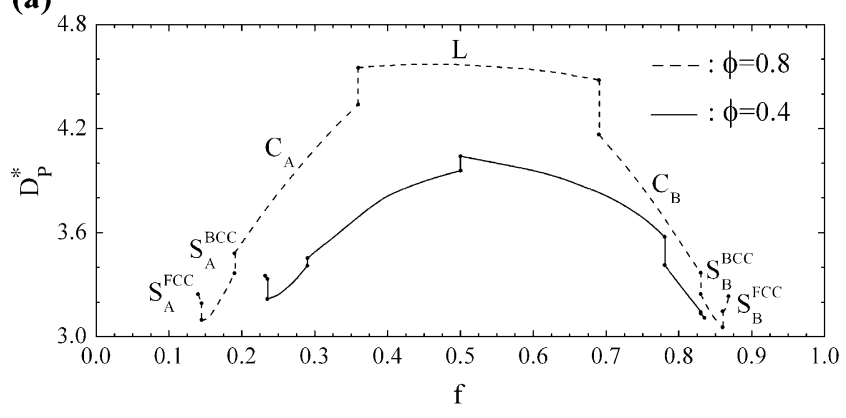

(b)

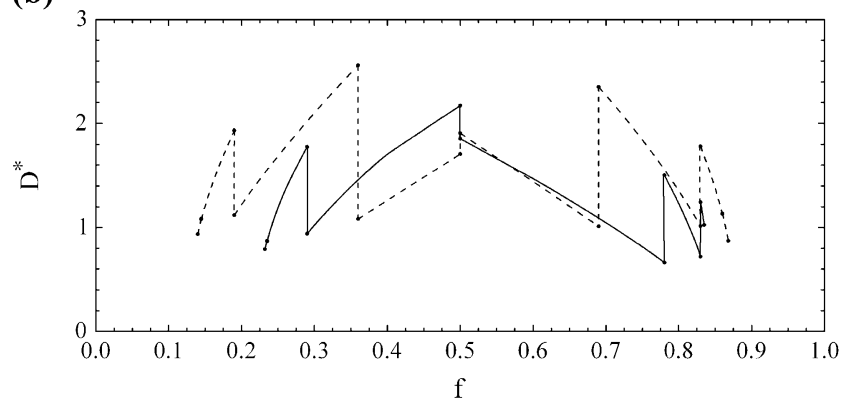

(c)

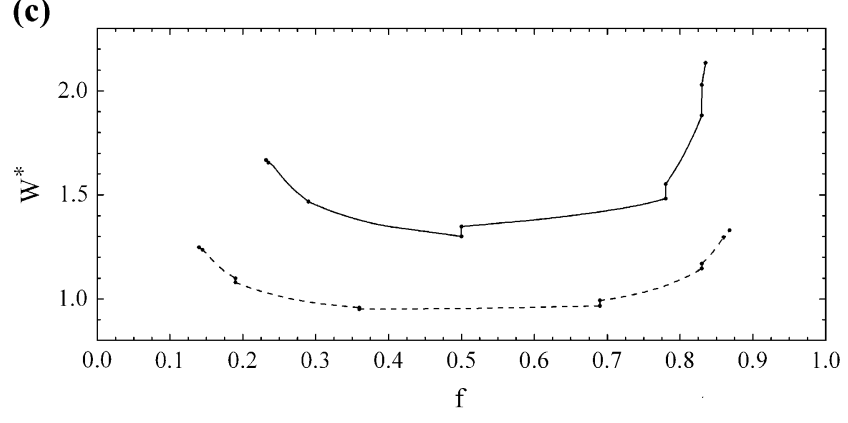

(d)

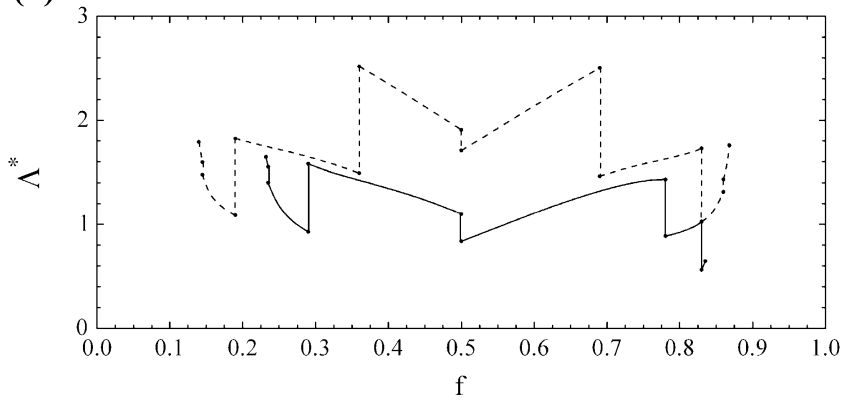

Fig. 7. Variation in (a) characteristic domain spacing $D_{\mathrm{P}}^{*}$, (b) minor-domain width $D^{*}$, (c) interfacial width $W^{*}$, and (d) major-domain width $\Lambda^{*}$, with changes in composition $f$ for a diblock copolymer with $N=150, \chi_{\mathrm{AB}} N=$ 40 in the presence of a B-selective solvent $\mathrm{S}\left(\chi_{\mathrm{AS}}=0.7, \chi_{\mathrm{BS}}=0.4\right)$ for $\phi=0.8$ and 0.4 .

in the characteristic domain spacing $D_{\mathrm{P}}^{*}$ of the spheres. This increasing behavior of $D_{\mathrm{P}}^{*}$ near the ODT, which has been observed in the diblock copolymer melts [35] and neutral diblock copolymer solutions [12], is mainly attributed to the fact that many of the minority blocks are pulled from the spherical domains and swell the matrix, i.e., $\Lambda^{*}$ increases. Further, $D_{\mathrm{P}}^{*}$ decreases discontinuously when the systems transform to a more curved microstructure, which results in allowing the larger blocks to relax at the expense of the smaller blocks, i.e., the major-domain width $\Lambda^{*}$ decreases while the minor-domain width $D^{*}$ increases discontinuously. When the systems undergo a transition from $\mathrm{S}^{\mathrm{BCC}}$ to $\mathrm{S}^{\mathrm{FCC}}$ by decreasing the composition of unfavorable blocks $f$, as expected, the spherical diameter $D^{*}$ decreases continuously, and both $\Lambda^{*}$ and $D_{\mathrm{P}}^{*}$ show a discontinuously increasing behavior. Note that a slightly discontinuous behavior of both $D^{*}$ and $\Lambda^{*}$ occurs at $f=0.5$ even within the same $\mathrm{L}$ phase, which is simply due to the definitions of $D^{*}$ and $\Lambda^{*}$ we choose. Recall that $D^{*}$ and $\Lambda^{*}$ in the $\mathrm{L}$ phase are defined as the domain width formed by the minority blocks and majority blocks, respectively. Hence, $D^{*}$ corresponds to the A-rich domain width when $f<0.5$ and the B-rich domain width when $f>0.5$. When more solvents are added $(\phi=0.4)$, though the variation of each length parameter with $f$ is similar to that for concentrated solutions, it is no longer symmetric about $f=0.5$. This is not surprising due to the more significant solvent selectivity at less concentrated solutions.

To analyze the effects of solvent selectivity on the variation of each domain length parameter with $\phi$ for each ordered phase, we choose a symmetric diblock copolymer with $N=150$ and $\chi_{\mathrm{AB}} N=40$ in the presence of a B-selective solvent and vary $\chi_{\mathrm{AS}} \geqq \chi_{\mathrm{BS}}=0.4$. As such, various ordered structures including $\mathrm{L}, \mathrm{C}_{\mathrm{A}}, \mathrm{S}_{\mathrm{A}}^{\mathrm{BCC}}$, and $\mathrm{S}_{\mathrm{A}}^{\mathrm{FCC}}$, are observed, as presented in Fig. 4(b). The resulting plot of characteristic domain spacing $D_{\mathrm{P}}^{*}$, minor-domain (i.e., A-rich domain) width $D^{*}$, interfacial width $W^{*}$, and major-domain (B-rich domain) width $\Lambda^{*}$ versus $\phi$ is shown in Fig. 8(a)-(d), respectively. When the solvent is neutral or slightly selective so that the added solvent causes a dilution effect, both domain lengths $D^{*}$ and $\Lambda^{*}$ show a decreasing behavior, and the interfacial width $W$ increases as $\phi$ decreases. As a result, the characteristic domain spacing $D_{\mathrm{P}}^{*}$ decreases as $\phi$ decreases. Upon increasing the solvent selectivity, this increasing trend of the interfacial width $W^{*}$ with the addition of solvent slows down indicating that the segregation degree between $\mathrm{A}$ and $\mathrm{B} / \mathrm{S}$ increases. That is, in order to minimize the contacts between $\mathrm{A}$ and $\mathrm{S}$, the interfacial area per chain decreases and thereafter the chain stretching normal to the interface increases with increasing the solvent selectivity. This implies that both the A-rich domain width $D^{*}$ and B-rich domain width $\Lambda^{*}$ increase with solvent selectivity. However, the fact that more solvents are expelled from the A-rich domains into the B-rich domains upon increasing the solvent selectivity may cause a further swelling of $\Lambda^{*}$ but a shrinking of $D^{*}$. As such, we observe that $D^{*}$ slightly reduces, but $\Lambda^{*}$ increases with solvent selectivity in the L phase. While for the cylindrical and spherical phases, $D^{*}$ shows a significantly increasing behavior upon increasing the solvent selectivity. Though the variation of the A-rich domain width $D^{*}$ with the solvent selectivity behaves differently for the $\mathrm{L}$ phase and the cylindrical/spherical phases, the characteristic domain spacing $D_{\mathrm{P}}^{*}$ within each phase shows an increasing behavior with the solvent selectivity. When the solvent selectivity becomes large, due to the fact that the added solvent strongly partitions into the matrix 
(a)

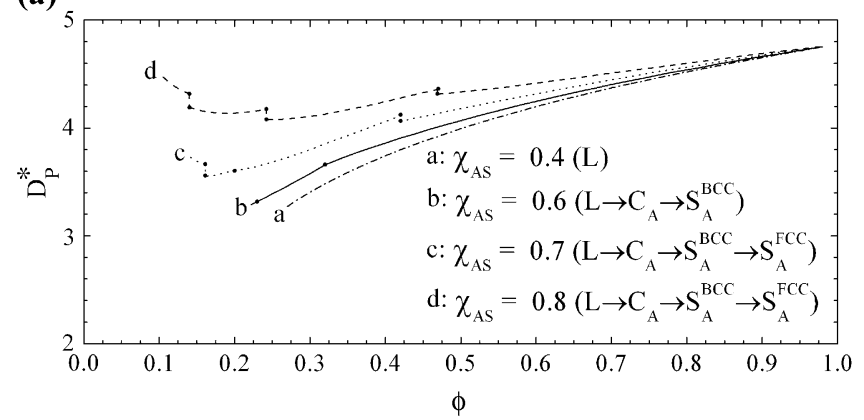

(b)

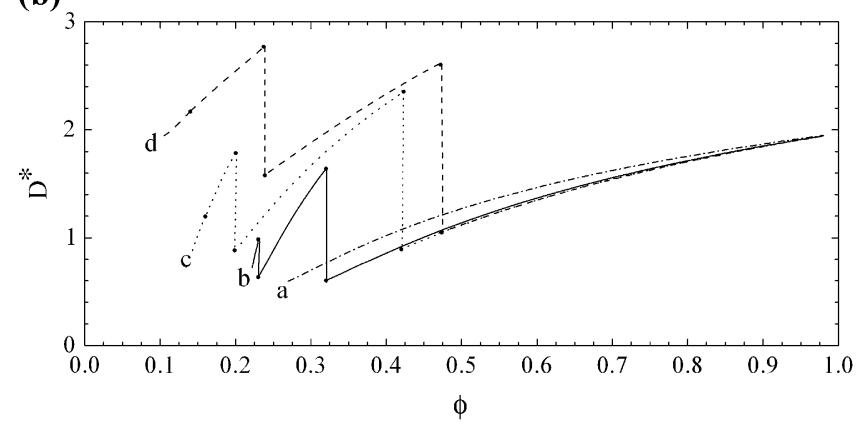

(c)

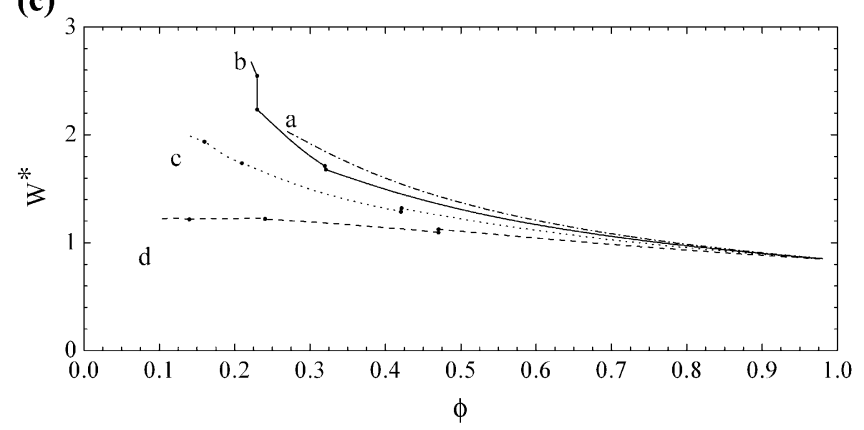

(d)

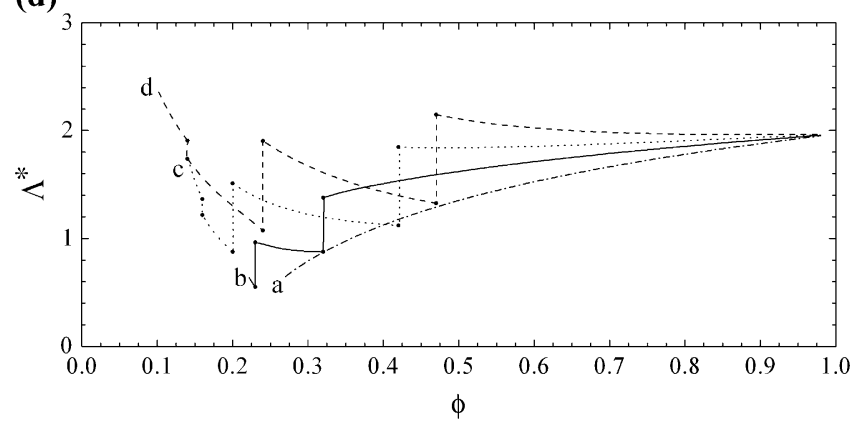

Fig. 8. Plot of (a) characteristic domain spacing $D_{\mathrm{P}}^{*}$, (b) minor-domain width $D^{*}$, (c) interfacial width $W^{*}$, and (d) major-domain width $\Lambda^{*}$, versus copolymer volume fraction $\phi$ for a symmetric diblock copolymer $(f=0.5)$ with $N=150$ and $\chi_{\mathrm{AB}} N=40$ in the presence of a B-selective solvent $\mathrm{S}$ at a fixed value of $\chi_{\mathrm{BS}}=0.4$ and various values of $\chi_{\mathrm{AS}}$.

regimes (B-rich domains), $\Lambda^{*}$ increases with increasing the solvent amount. Accordingly, we observe that the decreasing trend in $D_{\mathrm{P}}^{*}$ as $\phi$ decreases gets smoothened. As the solvent selectivity increases further, this increasing degree of $\Lambda^{*}$ even overcomes the decreasing degree of $D^{*}$, and thus the characteristic domain spacing $D_{\mathrm{P}}^{*}$ shows an increasing behavior upon increasing the solvent amount. This crossover behavior of domain spacing from decreasing with added solvent to increasing by increasing the solvent selectivity has also been captured in our previous SCMF analysis of the domain spacing within the same lamellar phase [15].

Indeed, the significant increase of $D^{*}$ upon increasing the solvent selectivity for the cylindrical and spherical phases is mainly attributed to the fact that more free chains remaining in the matrix and/or the interface are driven to aggregate into the cylinders or spheres. This can be clearly seen in Fig. 9, where we present the fraction of each component A, $\mathrm{B}$, and $\mathrm{S}$ into the A-rich (minor) domains, interface, and the matrix (B-rich domains), respectively, as a function of $\phi$ at a fixed $\chi_{\mathrm{BS}}=0.4$ and various values of $\chi_{\mathrm{AS}}$ for symmetric diblock copolymers $(f=0.5)$ with $N=150$ at $\chi_{\mathrm{AB}} N=40$. For a melt, around $80 \% \mathrm{~A}$ and $80 \% \mathrm{~B}$ segregate into the A-rich and B-rich domains, respectively, and the remaining $\mathrm{A}$ and $\mathrm{B}$ into the interface, as expected. When adding a B-selective solvent (decreasing $\phi$ ) and the solutions still maintain the stable lamellar phase, we find that increasing solvent selectivity for $\mathrm{B}$ enables more $\mathrm{B}$ in the interface to migrate into the B-rich domains, and solvent within the A-rich domains and interface into the B-rich domains. For component A, though the fraction of A in the A-rich domains $\tilde{\phi}_{\mathrm{A}}^{(D)}$ shows an increasing and $\tilde{\phi}_{\mathrm{A}}^{(W)}$ decreasing behavior with solvent selectivity, the variation of A fraction into each domain with $\phi$ is not affected much by the solvent selectivity. However, as $\phi$ keeps decreasing so that the systems form the cylindrical or spherical phase, the distribution of $\mathrm{A}$ is strongly influenced by the solvent selectivity. When the solvent is slightly selective $\left(\chi_{\mathrm{AS}}=0.6\right)$, both $\tilde{\phi}_{\mathrm{A}}^{(\Lambda)}$ and $\tilde{\phi}_{\mathrm{A}}^{(W)}$ exhibit a significantly increasing behavior with decreasing $\phi$, which is mainly attributed to the fraction of A in the A-rich domains pulled into the B-rich domains and interface. That is, there exist many free copolymer chains outside the cylindrical or spherical domains when the solvent is slightly selective. As the solvent selectivity increases, both the $\mathrm{A}$ fraction in the B-rich domains and the interface, $\tilde{\phi}_{\mathrm{A}}^{(\Lambda)}$ and $\tilde{\phi}_{\mathrm{A}}^{(W)}$, decrease, which thus causes an increase of the A fraction into the A-rich domains $\tilde{\phi}_{\mathrm{A}}^{(D)}$. For components $\mathrm{I}=\mathrm{B}$ and $\mathrm{S}$, we observe that the fraction into the B-rich domains $\tilde{\phi}_{\mathrm{I}}^{(\Lambda)}$ increases and the fraction along the interface $\tilde{\phi}_{\mathrm{I}}^{(W)}$ decreases with the solvent selectivity.

These results of each domain length as well as the distribution of each component into various segregated domains help to understand the transition of $\mathrm{S}^{\mathrm{BCC}} \rightarrow \mathrm{S}^{\mathrm{FCC}}$ upon increasing the solvent selectivity. Fig. 10 plots the schematic variation of the micelles from bcc to fcc array. For the spheres formed in the slightly selective solvent, though the solvent partitions preferentially to the matrix domains, there still remains a considerable amount of solvent inside the cores. Further, not $100 \%$ copolymer chains aggregate to form the micelles. Consequently, the intermicellar interactions become softer and thus these spheres adopt a bcc array. Upon increasing the solvent selectivity, though more solvents are expelled from the core which may cause a decrease in the micellar diameter, we find a significant increase in the spherical diameter as 

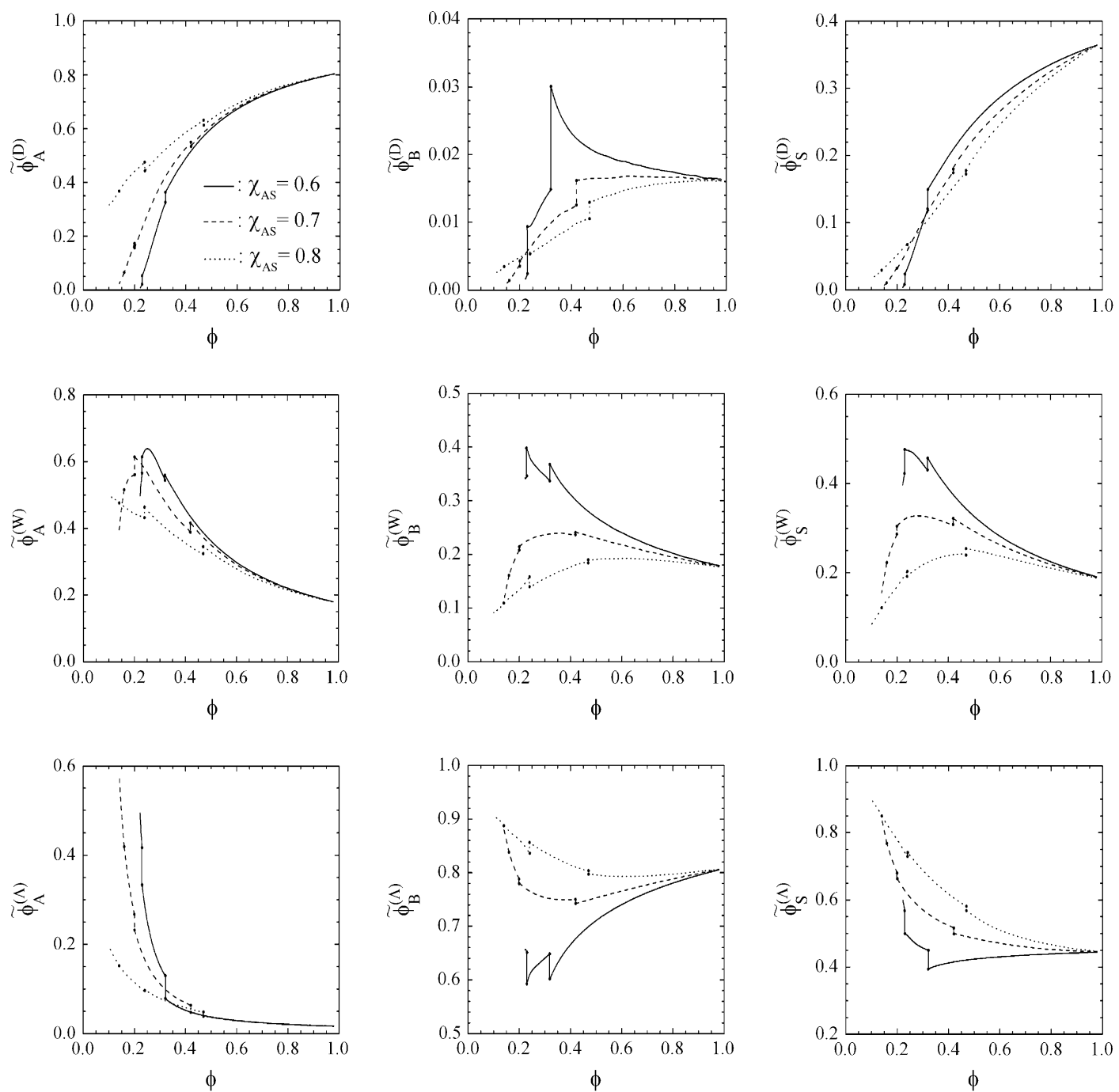

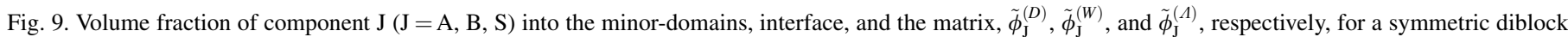
copolymer $(f=0.5)$ with $N=150$ and $\chi_{\mathrm{AB}} N=40$ in the presence of a B-selective solvent $\mathrm{S}$ at a fixed value of $\chi_{\mathrm{BS}}=0.4$ and various values of $\chi_{\mathrm{AS}}$.

more free chains remaining in the matrix and/or the interface are driven to aggregate into the spheres. As a result, these formed spheres become more impenetrable and adopt an fcc lattice. Though Lodge et al. [28] also proposed similar mechanisms, they reported a decrease in the spherical diameter upon increasing the solvent selectivity, which however, conflicts with our current results.

Increasing the solvent selectivity not only has a great influence on the concentration dependence of the characteristic domain spacing $D_{\mathrm{P}}^{*}$ but also the variation of $D_{\mathrm{P}}^{*}$ across the order-order transitions. As can be seen in Fig. 8(a), unlike the discontinuously decreasing behavior of $D_{\mathrm{P}}^{*}$ observed in the melts, $D_{\mathrm{P}}^{*}$ shows a continuous behavior across the $\mathrm{L} \rightarrow$ $\mathrm{C}_{\mathrm{A}}$ and $\mathrm{C}_{\mathrm{A}} \rightarrow \mathrm{S}_{\mathrm{A}}^{\mathrm{BCC}}$ transitions at $\chi_{\mathrm{AS}}=0.6$. When $\chi_{\mathrm{AS}}$ increases to 0.8 , each transition to a more curved structure even produces a discontinuous increase in $D_{\mathrm{P}}^{*}$. This behavior reflects that the increasing degree of the minor-domain (Arich domain) width $D^{*}$ across the transition to a more curved structure becomes more significant with increasing the solvent selectivity for B (Fig. 8(b)), which even overcomes the decreasing degree of the major-domain (B-rich) width $\Lambda^{*}$. As a result, when the systems transform to a microstructure with a larger curvature, $D_{\mathrm{P}}^{*}$ shows a continuous or even discontinuously increasing behavior as solvent selectivity increases. Further, the variation behavior in $D_{\mathrm{P}}^{*}$ across the transitions is also dependent of the copolymer composition $f$. Fig. 11(a)(d) presents the resulting $D_{\mathrm{P}}^{*}, D^{*}, W^{*}$, and $\Lambda^{*}$, as a function of $\phi$ for a diblock copolymer with $N=150, \chi_{\mathrm{AB}} N=40$, and various values of $f$, in the presence of a B-selective solvent $\left(\chi_{\mathrm{AS}}=0.7, \chi_{\mathrm{BS}}=0.4\right)$. Here we only present the results for $\mathrm{C}_{\mathrm{A}}, \mathrm{S}_{\mathrm{A}}^{\mathrm{BCC}}$, and $\mathrm{S}_{\mathrm{A}}^{\mathrm{FCC}}$. When $f=0.4$ such that the cylindrical and spherical structures are normal, $D_{\mathrm{P}}^{*}$ is continuous across the $\mathrm{C}_{\mathrm{A}} \rightarrow \mathrm{S}_{\mathrm{A}}^{\mathrm{BCC}}$ transition. As $f$ increases to greater than 0.5 so that the formed microstructures become inverted, $D_{\mathrm{P}}^{*}$ exhibits a discontinuously increasing behavior when $\mathrm{C}_{\mathrm{A}}$ transforms to $\mathrm{S}_{\mathrm{A}}^{\mathrm{BCC}}$ at the same solvent selectivity. This reveals that the larger the fraction of the unfavorable A-blocks for the solvent, the greater the chain stretching degree for the A-blocks in the minor-domains, which even overcomes the relaxing degree of the B-blocks in the matrix when the systems 


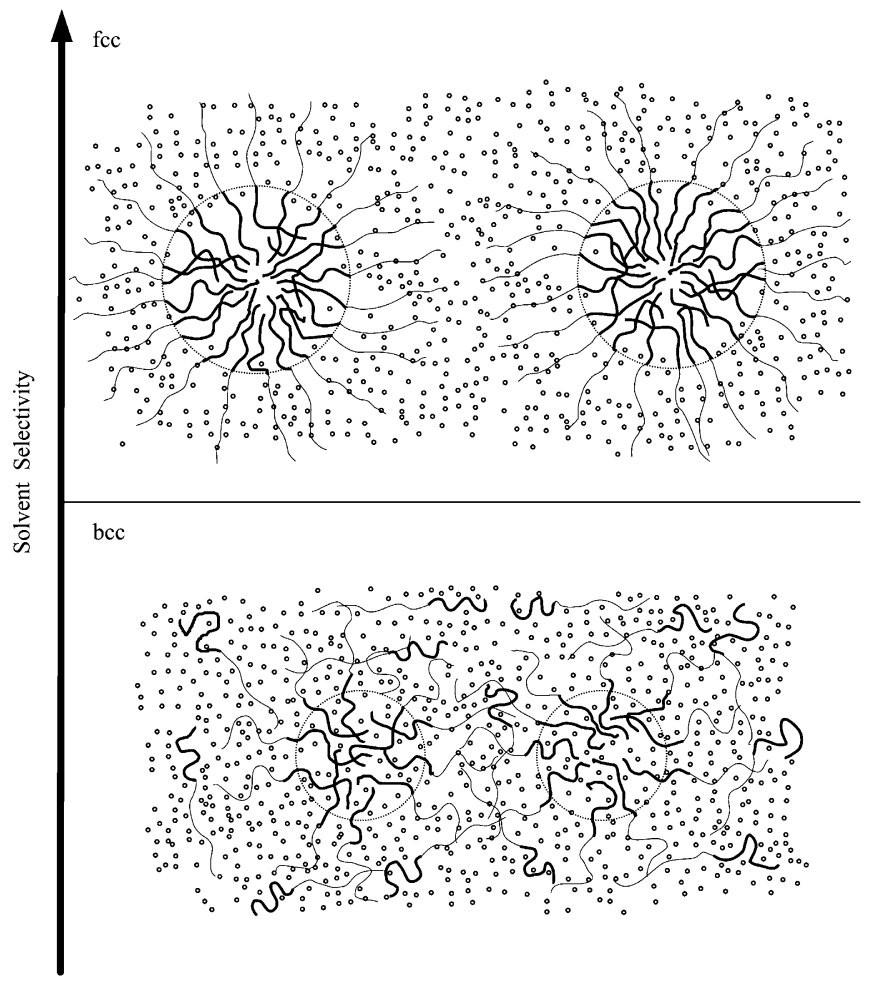

Fig. 10. Schematic variation of the ordered micelles from a thermotropic transition of bcc to fcc.

transform to a more curved structure. In addition, as $f$ increases, due to the fact that the B-blocks in the matrix are shorter and thus the swelling degree of the B-rich domains by the addition of a B-selective solvent increases, the increasing behavior of $\Lambda^{*}$ with increasing the solvent amount becomes more evident. As such, the increasing behavior of the characteristic domain spacing $D_{\mathrm{P}}^{*}$ by increasing the solvent amount is expected to occur at smaller solvent selectivity upon formation of the inverted structures.

\section{Conclusions}

We study the phase behavior as well as the microstructural domain sizes for an $\mathrm{AB}$ diblock copolymer in the presence of a selective solvent by self-consistent mean-field (SCMF) theory. In addition to the lamellae (L), hexagonally packed cylinders $(\mathrm{C})$, and body-centered cubic spheres $\left(\mathrm{S}^{\mathrm{BCC}}\right)$, we also consider face-centered cubic spheres $\left(\mathrm{S}^{\mathrm{FCC}}\right)$, which has been ignored in our previous work [7] but frequently observed in experiments $[15,21,24-30]$.

In examining the addition of a selective solvent on the resulting phase behavior, we focus on the formation of fcc and bcc packed spheres. In general, upon dilution of a selective solvent for the majority blocks so that the so-called "normal" spheres are formed by the minority blocks, due to the fact that the entropic penalty for larger corona layers is lower in bcc; bcc ordering is favored over fcc. It is interesting to find that the $S^{\mathrm{FCC}}$ regime for the normal spheres between $S^{\mathrm{BCC}}$ and D gets significantly broadened at lower values of $\phi$ with (a)

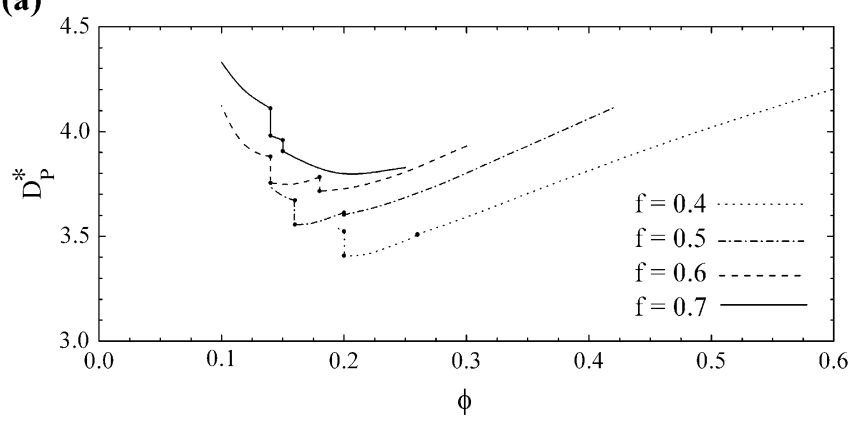

(b)

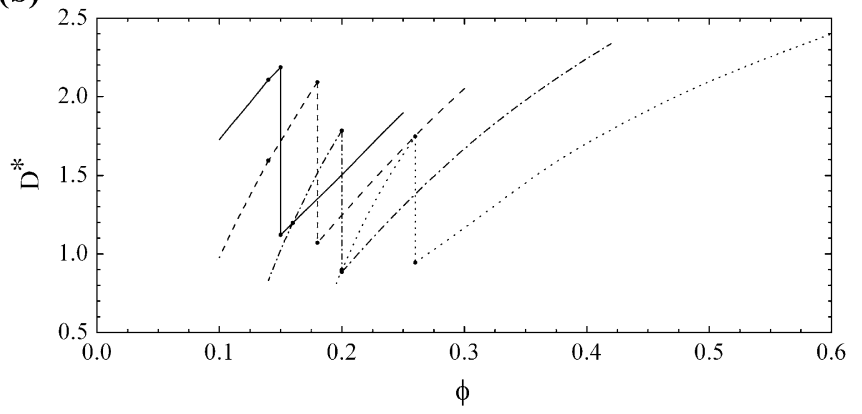

(c)

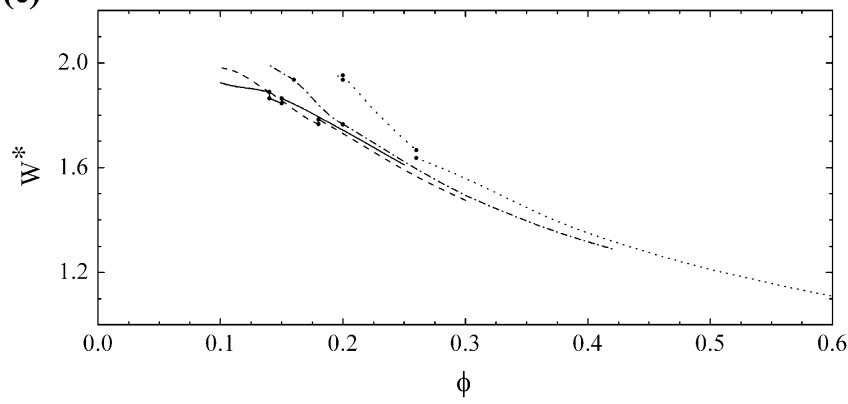

(d)

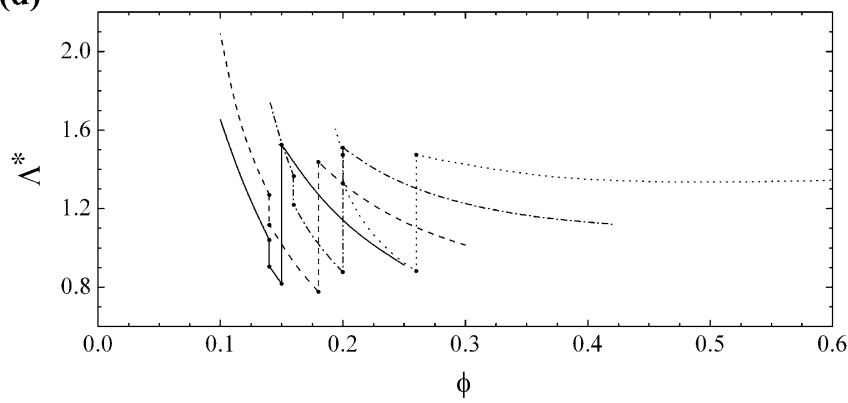

Fig. 11. Plot of (a) characteristic domain spacing $D_{\mathrm{P}}^{*}$, (b) minor-domain width $D^{*}$, (c) interfacial width $W^{*}$, and (d) major-domain width $\Lambda^{*}$, versus copolymer volume fraction $\phi$ for a diblock copolymer with $N=150, \chi_{\mathrm{AB}} N=40$, and various values of $f$, in the presence of a B-selective solvent $\left(\chi_{\mathrm{AS}}=0.7, \chi_{\mathrm{BS}}=0.4\right)$.

increasing the solvent selectivity. However, this has not been compared with the possible state of the disordered micelles. In the selective solvents for the minority blocks, i.e., the spheres are formed by the majority blocks, since more solvents are expelled into the matrix domains upon increasing the solvent selectivity and/or solvent amount, a greater degree of relieving the packing frustration for the blocks in the matrix 
is expected. In addition, the corona layers become thinner. Accordingly, these inverted spheres prefer a more dense fcc instead of a bcc packing upon increasing the solvent selectivity and/or solvent amount. Further, we observe that this thermotropic transition of bcc $\rightarrow \mathrm{fcc}$ is induced by the fact that the intermicellar interactions vary from long-range to short-range via a combination of the solvent exclusion from the cores and an increase in the aggregation number.

In the analysis of the microstructural length scales within each ordered phase when a solvent is added, the SCMF results have successfully captured the crossover behavior of characteristic domain spacing from decreasing with added solvent to increasing by increasing the solvent selectivity. With increasing the solvent selectivity, we observe that the interfacial width decreases, and both the major-domain width and the characteristic domain spacing within the same phase increase. However, the minor-domain width slightly reduces in the lamellar phase upon increasing the solvent selectivity. This is due to the fact that more solvent are expelled from the minordomains (rich in the unfavorable blocks) into the majordomains (rich in the favorable blocks) upon increasing the solvent selectivity, which thus causes a decrease of the minordomain width. While for the cylindrical and spherical phases, the minor-domain width shows a significantly increasing behavior upon increasing the solvent selectivity, which is mainly attributed to the fact that increasing solvent selectivity enables more free chains remaining in the matrix and/or the interface to aggregate into the cylinders or spheres. Varying the solvent selectivity not only has a great influence on the domain lengths within the same ordered phase but also across the order-order transitions. We find that upon increasing the solvent selectivity and/or the fraction of the unfavorable blocks, the variation of the characteristic domain spacing when the systems transform to a more curved structure changes from a discontinuous decreasing to even a discontinuous increasing behavior.

\section{Acknowledgements}

This work was supported by the National Science Council of the Republic of China through grant NSC 94-2216-E-002027.

\section{References}

[1] Lodge TP. Macromol Chem Phys 2003;204:265.

[2] Hadjichristidis N, Pispas S, Floudas G. Block copolymers: synthetic strategies, physical properties, and applications. New Jersey: Wiley; 2003.

[3] Hamley IW. The physics of block copolymers. New York: Oxford University Press; 1998.

[4] Hong KM, Noolandi J. Macromolecules 1983;16:1083.

[5] Whitmore MD, Noolandi J. J Chem Phys 1990;93:2946.

[6] Whitmore MD, Vavasour JD. Macromolecules 1992;25:2041.

[7] Huang CI, Lodge TP. Macromolecules 1998;31:3556.

[8] Helfand E, Tagami Y. J Chem Phys 1972;56:3592.

[9] Olvera de la Cruz M. J Chem Phys 1989;90:1995.

[10] Fredrickson GH, Leibler L. Macromolecules 1989;22:1238.

[11] Naughton JR, Matsen MW. Macromolecules 2002;35:5688.

[12] Chang Y, Hsueh HY, Chen WC, Huang CI. Polymer 2005;46:3942.

[13] Lodge TP, Pan C, Jin X, Liu Z, Zhao J, Maurer WW, et al. J Polym Sci Part B Polym Phys 1995;33:2289.

[14] Hanley KJ, Lodge TP. J Polym Sci Part B Polym Phys 1998;36:3101.

[15] Hanley KJ, Lodge TP, Huang CI. Macromolecules 2000;33:5918.

[16] Lodge TP, Hanley KJ, Pudil B, Alahapperuma V. Macromolecules 2003; $36: 816$.

[17] Hashimoto T, Shibayama M, Kawai H. Macromolecules 1983;16:1093.

[18] Shibayama M, Hashimoto T, Hasegawa H, Kawai H. Macromolecules 1983;16:1427.

[19] Watanabe H, Kotaka T, Hashimoto T, Shibayama M, Kawai H. J Rheol 1982;26:153

[20] Shibayama M, Hashimoto T, Kawai H. Macromolecules 1983;16:16.

[21] McConnell GA, Gast AP, Huang JS, Smith SD. Phys Rev Lett 1993;71: 2102.

[22] McConnell GA, Lin MY, Gast AP. Macromolecules 1995;28:6754.

[23] McConnell GA, Gast AP. Phys Rev E 1996;54:5447.

[24] McConnell GA, Gast AP. Macromolecules 1997;30:435.

[25] Pople JA, Hamley IW, Fairclough JPA, Ryan AJ, Komanschek BU, Gleeson AJ, et al. Macromolecules 1997;30:5721.

[26] Hamley IW, Pople JA, Diat O. Colloid Polym Sci 1998;276:446.

[27] Lodge TP, Pudil B, Hanley KJ. Macromolecules 2002;35:4707.

[28] Bang J, Lodge TP, Wang X, Brinker KL, Burghardt WR. Phys Rev Lett 2002;89:215505

[29] Bang J, Lodge TP. J Phys Chem B 2003;107:12071.

[30] Park MJ, Bang J, Harada T, Char K, Lodge TP. Macromolecules 2004;37: 9064.

[31] Lai C, Russel WB, Register RA. Macromolecules 2002;35:841.

[32] Lai C, Russel WB, Register RA. Macromolecules 2002;35:4044.

[33] Banaszak M, Whitmore MD. Macromolecules 1992;25:3046.

[34] Noolandi J, Shi AC, Linse P. Macromolecules 1996;29:5907.

[35] Matsen MW, Bates FS. J Chem Phys 1997;106:2436.

[36] Henry NFM, Lonsdale K, editors. International tables for X-ray crystallography. Birmingham: Kynoch; 1969.

[37] Dormidontova EE, Lodge TP. Macromolecules 2001;34:9143. 